\title{
Structural basis for tunable control of actin dynamics by myosin-15 in mechanosensory stereocilia
}

Rui Gong ${ }^{1}$, Fangfang Jiang ${ }^{2}$, Zane G. Moreland ${ }^{2}$, Matthew J. Reynolds ${ }^{1}$, Santiago Espinosa de los Reyes ${ }^{1}$, Pinar S. Gurel ${ }^{1}$, Arik Shams ${ }^{3}$, Michael R. Bowl ${ }^{4,5}$, Jonathan E. Bird ${ }^{2, *}$, Gregory M. Alushin ${ }^{1, * \dagger}$

${ }^{1}$ Laboratory of Structural Biophysics and Mechanobiology, The Rockefeller University, New York, NY, USA.

${ }^{2}$ Department of Pharmacology and Therapeutics, University of Florida, Gainesville, FL, USA.

${ }^{3}$ Laboratory of Molecular Genetics, National Institute on Deafness and Other Communication Disorders, National Institutes of Health, Bethesda, MD, USA.

${ }^{4}$ Mammalian Genetics Unit, MRC Harwell Institute, Harwell Campus, Oxfordshire, UK.

${ }^{5}$ UCL Ear Institute, University College London, London, UK.

*Correspondence: j.bird@ufl.edu; galushin@rockefeller.edu

${ }^{\dagger}$ Lead contact 


\section{Summary:}

The motor protein myosin-15 is necessary for the development and maintenance of mechanosensory stereocilia, and myosin-15 mutations cause profound deafness. In a companion study, we report that myosin-15 nucleates actin filament ("F-actin") assembly and identify a progressive hearing loss mutation (p.D1647G, "jordan") which disrupts stereocilia elongation by inhibiting actin polymerization. Here, we present cryo-EM structures of myosin-15 bound to F-actin, providing a framework for interpreting deafness mutations and their impacts on myosin-stimulated actin assembly. Rigor myosin-15 evokes conformational changes in F-actin yet maintains flexibility in actin's D-loop, which mediates inter-subunit contacts, while the jordan mutant locks the D-loop in a single conformation. ADP-bound myosin-15 also locks the D-loop, which correspondingly blunts actin-polymerization stimulation. We propose myosin-15 enhances polymerization by bridging actin protomers, regulating nucleation efficiency by modulating actin's structural plasticity in a myosin nucleotide-state dependent manner. This tunable regulation of actin polymerization could be harnessed to precisely control stereocilium height.

\section{$\underline{\text { Key words: }}$}

actin cytoskeleton, myosin motor proteins, cryo-electron microscopy, stereocilia, deafness, DFNB3, MYO15A 


\section{Introduction:}

The stereocilia of inner ear hair cells are rod-like membrane protrusions responsible for sound detection. Each hair cell assembles a staircase-shaped mechanosensory hair bundle during development, which contains three rows of stereocilia of increasing height. Stereocilia are primarily composed of parallel bundles of filamentous actin (F-actin) crosslinked to form a para-crystalline array (DeRosier et al., 1980; Tilney et al., 1980). Stereocilia develop from microvilli that undergo an exquisite morphogenetic program characterized by F-actin elongation and bundling, presumed to be orchestrated by coordinated actin polymerization and bundling protein engagement (Tilney et al., 1992). Once a stereocilium is established, slow actin dynamics maintain its architecture throughout adult life (Drummond et al., 2015; Narayanan et al., 2015; Zhang et al., 2012). Finely-tuned regulation of actin dynamics is critical for stereocilium height determination, and mutations in actin and its regulatory factors are frequently associated with hearing loss (Barr-Gillespie, 2015; Drummond et al., 2012). Nevertheless, the molecular mechanisms which precisely define the height of individual stereocilia during development and maintain them for a lifetime remain elusive.

Unconventional myosin-15 (encoded by the Myo15 gene in mouse, and MYO15A in humans) is a plus-end directed molecular motor essential for stereocilia development and maintenance (Anderson et al., 2000; Belyantseva et al., 2003; Belyantseva et al., 2005; Bird et al., 2014; Fang et al., 2015; Probst et al., 1998; Wang et al., 1998). Over 300 mutations have been mapped to the human MYO15A gene that cause autosomal recessive non-syndromic deafness, DNFB3 (Rehman et al., 2016; Wang et al., 1998). Within hair cells, myosin-15 localizes to the distal tips of stereocilia, the major sites of actin turnover which are enriched with F-actin plus ("barbed”) ends (Belyantseva et al., 2003; Belyantseva et al., 2005;

Drummond et al., 2015; Narayanan et al., 2015; Rzadzinska et al., 2004). In mice homozygous for the Myo15 "shaker 2" allele (p.C1779Y), myosin-15 trafficking is disrupted in nascent stereocilia

(Belyantseva 2003), resulting in failure of stereocilia to elongate and profound hearing loss (Probst et al., 1998). Myosin-15 transports whirlin (WHRN), EPS8, GPSM2, and GNAI3 to stereocilia tips, an “elongation network" of interacting proteins required for stereocilium biogenesis, each member of which 
phenocopies shaker 2 when individually mutated in mice (Belyantseva et al., 2005; Delprat et al., 2005;

Manor et al., 2011; Mauriac et al., 2017; Mburu et al., 2003; Tadenev et al., 2019; Tarchini et al., 2016;

Zampini et al., 2011). As EPS8 is a known actin-binding protein (ABP) which exhibits filament bundling and plus-end capping activity (Disanza et al., 2004; Hertzog et al., 2010), it has been proposed that myosin-15 mediates stereocilia growth by transporting elongation network components to the tip compartment, where they serve as the regulatory machinery governing F-actin assembly (Belyantseva et al., 2005; Delprat et al., 2005; Manor et al., 2011; Mauriac et al., 2017; Tadenev et al., 2019).

Nevertheless, the functional consequences of specific myosin-15 mutations remain poorly understood due to the absence of structural data and limited functional characterization of the myosin- 15 protein.

In a companion manuscript, we identified the myosin-15 missense mutation p.D1647G, hereafter referred to as the "jordan" mutant (Figure 1A), in a forward genetic screen for lesions leading to progressive hearing loss in mice (Moreland, 2021). The jordan mouse develops abnormally short stereocilia which retract during aging. Although its actin-activated ATPase activity and F-actin binding affinity are somewhat compromised, purified jordan myosin-15 is nevertheless an active motor, and WHRN, EPS8, GPSM2 and GNAI3 localize to the tips of developing stereocilia in the jordan mouse, leading us to hypothesize that myosin-15 could control stereocilium height through additional mechanisms. Consistently, we found that the motor-domain containing myosin-15 S1 fragment dramatically stimulates actin polymerization in vitro, with the nucleotide-free "rigor" state specifically acting as a nucleation factor, while the jordan mutant instead suppresses actin assembly (Moreland, 2021). Early studies of the S1 fragment of skeletal muscle myosin demonstrated its capacity to nucleate actin polymerization in vitro (Fievez et al., 1997; Lheureux et al., 1993; Miller et al., 1988), but a physiological role for this activity has not, to our knowledge, been reported. Our studies suggest myosin15 directly regulates actin dynamics in stereocilia, but the underlying molecular mechanism, and how it is disrupted by a single amino acid substitution in the jordan mutant, remain unknown.

F-actin assembly and disassembly are tightly regulated by dozens of ABPs, including severing proteins, capping proteins, and nucleators, whose activities must be precisely coordinated to construct 
elaborated cellular protrusions such as stereocilia (Pollard, 2016; Tilney et al., 1992). These ABPs frequently utilize their actin-binding interfaces to modulate G-actin monomer / F-actin protomer conformation and thereby fine-tune actin polymerization dynamics (Crevenna et al., 2015; Dominguez and Holmes, 2011; Pollard, 2016). The structurally polymorphic actin subdomain 2, or "D-loop", which mediates longitudinal interactions between F-actin protomers, plays a prominent role in this conformational regulation (Das et al., 2020; Durer et al., 2012; Galkin et al., 2010). Several myosin motors have been reported to modulate actin's conformation through direct contacts with the D-loop upon F-actin binding (Gurel et al., 2017; Mentes et al., 2018; von der Ecken et al., 2016), and distinct actin conformations were visualized at the Mg-ADP and rigor stages of myosin-6's mechanochemical cycle (Gurel et al., 2017). This led us to hypothesize that reciprocal conformational changes at the actin-myosin interface could be harnessed by myosin-15 during its mechanochemical cycle to precisely regulate F-actin polymerization dynamics to control stereocilium height.

Here we report the cryo-electron microscopy (cryo-EM) structure of the rigor wild-type myosin15 motor domain bound to F-actin at $2.84 \AA$ resolution, to our knowledge the highest-resolution actomyosin structure to date, providing a framework for broadly interpreting deafness mutations in the motor domain and at the actin-myosin interface. To probe the mechanisms by which myosin-15 regulates F-actin assembly, we also obtained cryo-EM structures of F-actin alone (2.82 $\AA$ ), rigor jordan myosin-15 (3.76 ̊), and Mg-ADP wild-type myosin-15 (3.63 $\AA$ ) bound to F-actin. The unprecedented resolution of our F-actin alone structure reveals a region of the D-loop (residues G48-Q49) that is structurally flexible but nevertheless primarily adopts a mixture of two conformations. Rigor wild-type myosin-15 engagement elicits rearrangements at the actin-myosin interface which are propagated through the actin structure, yet the D-loop continues to adopt two conformations. Binding of the jordan mutant evokes similar overall rearrangements but strikingly locks the D-loop in a single conformation, as does Mg-ADP wild-type myosin-15, which shows reduced F-actin stimulation relative to the rigor state in polymerization assays. Our studies suggest strongly-bound myosin-15 promotes F-actin assembly by reinforcing longitudinal contacts between actin protomers with a nucleation efficiency controlled by the 
motor domain's nucleotide-state, which modulates actin conformational flexibility mediating F-actin

polymerization. We propose this tunable direct regulation of actin assembly dynamics facilitates precise control of stereocilia height by myosin-15 in vivo, which is completely compromised by the jordan deafness mutation.

\section{Results:}

\section{Cryo-EM reconstructions of actomyosin-15 complexes and bare F-actin}

Using cryo-EM and the iterative-real space helical symmetry reconstruction (IHRSR) procedure as implemented in RELION-3 (Egelman, 2007; Zivanov et al., 2018) we determined structures of a wildtype mouse myosin-15 construct containing the motor domain and 2 IQ motifs (Bird et al., 2014) ("MD2IQ”, Figure 1A) bound to F-actin at a 1:1 stoichiometry in the rigor and Mg-ADP states at an average resolution of $2.84 \AA$ and $3.63 \AA$, respectively (STAR Methods). We additionally determined the structure of the jordan deafness mutant bound to F-actin at $3.76 \AA$ resolution (Figures 1B, S1A,B). Our rigor wildtype structure represents a substantial improvement in resolution over previously reported actomyosin reconstructions (Gurel et al., 2017; Mentes et al., 2018; Risi et al., 2021; von der Ecken et al., 2016), facilitating building an accurate atomic model of the myosin-15 motor domain (Figure 1C), which has not, to our knowledge, previously been structurally visualized in the absence or presence of F-actin.

As has been reported in other cryo-EM studies of actomyosin (Gurel et al., 2017; Mentes et al., 2018; Risi et al., 2021; von der Ecken et al., 2016), the local resolution of these reconstructions is highest in the center of the filament and decreases radially outwards towards the distal tip of the myosin lever arm (Figure S1B). For all three complexes, the resolution of F-actin and the actin-myosin interface extends beyond $3 \AA$, yielding models with unambiguous side-chain density within these areas (Figures 1D,E; S1B). Most side chains in the motor domain are clearly resolved and secondary structures can be identified in and around the distal converter domain (Figures 1E, S1B). To visualize structural changes evoked in F-actin by myosin-15, we also reconstructed bare F-actin at a global resolution of $2.82 \AA$ (Figures 1B; S1A,B), to our knowledge the highest resolution mammalian F-actin structure reported to 
date. This density map facilitated direct model building and refinement of actin's bound Mg-ADP and all actin residues except the first five at the protein's flexible $\mathrm{N}$ terminus.

\section{Structural interpretation of deafness-causing myosin-15 mutations}

Within the motor domain of myosin-15, a total of 59 missense mutations targeting 58 residues have been identified in DFNB3 patients (Rehman et al., 2016; Zhang et al., 2019). Of these 58 residues, 2 are located in disordered regions which were not resolved in our reconstruction: the remaining 56 can be mapped onto the structure (Figure 2A). Sequence alignment of the mouse and human myosin-15 motor domains shows 54 of the 56 residues are identical and 2 of them are highly similar (Figure S2). Examining the distribution of these deafness-causing mutations leads us to classify them into four groups

(Figure 2A: unless otherwise noted, residues cited correspond to the human protein). Mutations in the first group are found in the core of the motor domain and are likely to disrupt proper folding and thereby completely compromise function. For example, the p.S1465P mutation introduces a proline in the middle of a long helix and the p.A1532T/p.A1535D mutations substitute alanine with a polar or charged amino acid in the hydrophobic core (Figure 2B), all of which we anticipate will severely impede folding.

This group additionally includes mouse C1779, the site of the pathogenic p.C1779Y deafness mutation in the shaker 2 allele, contrary to its originally predicted location at an actin-binding interface (Probst et al., 1998). This residue sits in a hydrophobic core surrounded by mouse I1297, L1275, A1308 and V1777 (Figure S3A), where substitution with a bulky hydrophilic tyrosine would be anticipated to disrupt the proper folding of the transducer. Consistent with this interpretation, recombinantly expressed shaker 2 (p.C1779Y) motor domain is a soluble aggregate when purified and subjected to size exclusion chromatography (Moreland, 2021). Furthermore, the human counterpart (A1324) of mouse A1308, which is adjacent to $\mathrm{C} 1779$ (Figure S3A), was also found to be substituted with the highly polar residue aspartic acid in a pedigree with profound deafness (Zhang et al., 2018), consistent with disruption of the hydrophobic core resulting in misfolding and aggregation. 
Mutations in the second group are located in regions mediating interactions between the motor's subdomains, which are likely to interfere with coordinated rearrangements required to execute the ATPase mechanochemical cycle (Houdusse and Sweeney, 2016) The side chain of the highly conserved K1541 from the upper 50-kDa subdomain forms a hydrogen bond with the backbone of H1704 in the loop connecting the upper 50-kDa and lower 50-kDa subdomains (Figure 2C and Figure S2). This interaction likely plays a stabilizing role in the cleft, and the charge inversion p.K1541E mutation is anticipated to disrupt it and hinder cleft closure. The third group are located in consensus ATPase motifs, including switch I, switch II, and the P-loop. These mutations are expected to have a deleterious impact on the mechanochemical cycle by impeding ATP binding and/or hydrolysis (Figure 2D and Figure S2). Finally, a fourth group of mutations are distributed in solvent exposed loop regions with no obvious pattern (Figure 2A). We speculate these residues mediate binding contacts with yet to be identified partner proteins or intramolecular contacts involved in regulating myosin-15's motor function, which could include an interface between the motor domain and the large $133 \mathrm{kDa}$-terminal domain of myosin- 15 isoform 1 (MYO15-1; Figure 1A) that is required for postnatal stereocilia maintenance (Fang et al., 2015). Our structural mapping analysis suggests that mutations in myosin-15 cause pathology by either interfering with canonical myosin-15 ATPase motor activity, or with non-canonical functions such as mediating protein-protein interactions at the stereocilia tips.

\section{A structurally diversified myosin-15-actin interface}

We next undertook a detailed analysis of the myosin-15-F-actin interface to identify specific contacts which could contribute to myosin-15's F-actin nucleation activity. We also examined the interface for contacts which might be impacted by deafness mutations in both myosin-15 and $\gamma$-actin (encoded by the ACTG1 gene in humans), one of the primary actin isoforms expressed in hair cells (Figure S3B). Like other myosins, myosin-15 employs conserved structural elements to interact with Factin: the Helix-Loop-Helix (HLH) motif, the cardiomyopathy (CM) loop, loop 2, loop 3, and the activation loop (Figure 3A and S4A). However, residue-level interactions have diversified among 
myosins to fine-tune the functions of superfamily members (Gurel et al., 2017; Mentes et al., 2018; von der Ecken et al., 2016). Myosin-15's HLH motif (I1641-P1671) mediates the major contact with F-actin through its engagement with two longitudinally adjacent actin subunits (which we here refer to as subunits "i”" and " $i+2$ ") along a protofilament (Figure 3A). The first helix of the HLH includes myosin15 residue D1647, the site of the p.D1647G mutation in the jordan allele, which forms hydrogen bonds with S350 and T351 on subdomain 1 of subunit i (Figure 3B). Notably, D1647 is highly conserved among myosin supefamily members, indicating this interaction is likely broadly important for myosin function (Figure S2). Mutation of this site in myosin-2 resulted in a ten-fold reduction of F-actin binding affinity (Furch et al., 2000), and the F-actin binding of jordan mutant myosin-15 is also substantially decreased (Moreland, 2021).

In the loop portion of the HLH, a pair of hydrophobic residues (F1651 and P1652) inserts into a hydrophobic pocket formed by subdomains 1 and 3 of actin subunit $i$ and the D-loop of subunit $i+2$ (Figure 3C), where L349 of subunit i closely engages with myosin-15 F1651. The $\gamma$-actin mutation L349M causes profound deafness in human patients (Sloan-Heggen et al., 2016), and we anticipate replacement of leucine by the bulkier methionine impairs myosin-15 engagement by preventing access to this pocket (Figure 3C and S3B). Additionally, Q1653 forms a hydrogen bond with the backbone of subunit i residue G146 (Figure S4B). The second helix engages with the N-terminal portion of subunit $\mathrm{i}+$ 2's D-loop through a network of electrostatic interactions. Residue K1662 of myosin-15 forms a hydrogen bond with the sidechain of D-loop residue Q49, and myosin-15 Y1665 also forms a possible N$\mathrm{H} \cdots \pi$ bond with this actin residue (Figure S4B).

The activation loop (I1634-G1640) preceding the HLH motif has been suggested to activate myosins through contacts with actin's negatively charged N terminus (Varkuti et al., 2012; Varkuti et al., 2015). Indeed, K1637 forms a possible salt bridge with subunit $i+2$ residue 4E, partially stabilizing actin's flexible $\mathrm{N}$ terminus (Figure $\mathrm{S} 4 \mathrm{C}$ ). This ionic interaction is present in both the Mg-ADP and rigor states (Figure S4C), indicating that it is likely involved in the initial weak-to-strong F-actin binding transition in the case of myosin-15. Loop 2 has also been implicated in mediating initial contacts during 
the weakly-bound phase of the myosin mechanochemical cycle prior to phosphate release (Murphy and Spudich, 1999; Onishi et al., 2006; Uyeda et al., 1994). This region is largely disordered in our structures, suggesting loop 2 engagement is dispensable for strong binding of myosin-15 to F-actin. Discontinuous density is present for several residues extending from the C-terminal base of loop 2, which orients towards the $\mathrm{N}$ terminus of actin and may form weak interactions (Figure S4D). This is in striking contrast to non-muscle myosin-1c (hereafter "myosin-1") or non-muscle myosin-2c (hereafter "myosin-2"), where loop 2 is either partially or fully ordered at the strongly-bound interface (Mentes et al., 2018; von der Ecken et al., 2016).

The CM loop adopts a beta hairpin fold and packs tightly against subdomain 1 of subunit $\mathrm{i}$ (Figure 3A and 3D). In contrast to the CM loops of myosin-1 and myosin-2, which primarily engage in hydrophobic interactions with F-actin (Mentes et al., 2018; von der Ecken et al., 2016), myosin-15's CM loop-F-actin hydrophobic interface is minimal and is instead dominated by a network of electrostatic interactions. Specifically, the non-conserved residue R1520 at the tip of the CM loop likely mediates a myosin-15-specific salt bridge with actin D56. Similar to myosin-1, the highly conserved residue E1521 mediates electrostatic contacts with actin residues K336 and Y337. The side chain of T1525 also forms a hydrogen bond with actin E334, which is not observed for myosin-1 or myosin-2 (Figure 3D). Myosin-15 possesses a relatively short loop 3 which nevertheless mediates substantial contacts with subdomain 1 of subunit $\mathrm{i}+2$. Compared with myosin- 1 and myosin-2, myosin-15's loop 3 assumes a distinct conformation to form divergent interactions (Figure S4E). Notably, myosin-15 P1679 makes Van der Waals contacts with actin N92 and R95, and M1678 sits in a shallow hydrophobic groove cradled by actin Y91, V96, A97 and P98. This interface is further strengthened by a probable salt bridge between myosin K1677 and actin E99 (Figure 3E). Collectively, we observe numerous myosin-15-specific interactions with F-actin, consistent with specialization of this interface for myosin-15 function in stereocilia.

\section{Rigor myosin-15 evokes structural changes in F-actin while maintaining D-loop flexibility}


As rigor myosin-15 potently stimulates F-actin polymerization (Moreland, 2021), we next compared two longitudinally adjacent actin subunits from our F-actin alone and rigor wild-type actomyosin-15 structures, hypothesizing myosin-15 could regulate actin polymerization by modulating Factin conformation. Consistent with previously reported actomyosin structures (Gurel et al., 2017; Mentes et al., 2018; Risi et al., 2021; von der Ecken et al., 2016), the overall conformation of actin in the presence and absence of myosin-15 is highly similar, with a global RMSD of $0.51 \AA$ (Figure 4A). We primarily observe remodeling adjacent to the myosin- $15 \mathrm{HLH}$, which is propagated through the structure into a global compression of the filament along the longitudinal axis clearly visible in a morph between the density maps of bare F-actin and rigor actomyosin-15 (Video S1). Consistently, the helical rise decreases $0.12 \AA$ upon myosin-15 binding, with no substantial alteration of the helical twist (Figure S1C). The short $\alpha$-helix in actin subunit i which forms contacts with D1647 shifts slightly towards the minus end to mediate this compression (Figure 4B). This is accommodated by rearrangements in the D-loop of subunit $\mathrm{i}+2$, which is also engaged by the myosin- $15 \mathrm{HLH}$.

Several recent high-resolution cryo-EM studies of F-actin have indicated that the D-loop is structurally dynamic within the context of the filament (Chou and Pollard, 2019; Das et al., 2020; Merino et al., 2018; Pospich et al., 2020). At a high threshold (0.036 RMS) our bare F-actin map features ambiguity surrounding residues G48 and Q49, despite clear density for all other residues in the D-loop (Figure 4C). However, at a lower threshold (0.025 RMS), the unprecedented resolution of our map reveals two continuous paths for the backbone, allowing two distinct conformations of the D-loop to be modeled: an "In" conformation where the main-chain of G48 flips in towards the center of the filament and Q49 faces the solvent, and an "Out" conformation where the main-chain of G48 is exposed and Q49 is buried in the center of the D-loop (Figures 4C, S5). Based on the respective strengths of the 2 density paths, the "Out" conformation is predominant in the bare F-actin structure, likely stabilized by an interaction between the side chain of Q49 of subunit $i+2$ and the side chain of Y169 from the adjacent subunit i (Figure 4C). Upon rigor myosin-15 binding, the D-loop transitions to primarily being in the "In" state (Figure 4B,D), but displaced $2.7 \AA$ outward from the filament axis relative to the bare F-actin 
conformation (Figure 4E), enabling an interaction between myosin HLH residue Y1665 and the flippedout sidechain of actin D-loop residue Q49 (Figure 4D). Nevertheless, at a lower threshold (0.028 RMS), density resembling the "Out" conformation in bare actin is clearly visible (Figure 4D, Video S2), suggesting that rigor myosin-15 engages its binding site while maintaining F-actin's intrinsic structural plasticity in the D-loop.

\section{The myosin-15 jordan deafness mutant locks the D-loop in the "In" conformation}

To gain insight into structural mechanisms underlying the jordan mutant's suppression of actin assembly and disruption of stereocilia growth (Moreland, 2021), we next compared the rigor structures of wild-type and jordan mutant actomyosin-15. The overall conformations of the motor domains are essentially indistinguishable, arguing the jordan mutation does not grossly compromise myosin-15's structure (Figure S6A). The D1647G substitution abolishes the contacts formed between D1647 and actin residues S350 and T351 (Figure 5A) without disrupting the conformation and positioning of the HLH or discernibly perturbing any other contacts at the myosin-15-actin interface (Figure S6B-E). We next examined the structures for global differences in actin conformation, hypothesizing these could underlie the differential effects upon actin polymerization. Morphing the density maps reveals minimal changes, and the refined helical parameters of the reconstructions are nearly identical (Figure S1C and Video S3), indicating the jordan mutation does not substantially impact the global structural rearrangements in Factin evoked by myosin-15. Consistently, the short $\alpha$-helix comprising S350 and T351 of subunit i still undergoes a shift indistinguishable from that observed in the wild-type reconstruction (Figure 5B), indicating this conformational transition is not solely evoked by contacts formed with D1647 and is instead likely an allosteric effect driven by HLH engagement. Collectively, these data suggest that the jordan mutation also does not grossly disrupt the actin-myosin-15 interface or myosin-15's conformational regulation of F-actin.

We thus turned to an alternative hypothesis, that the jordan mutant could disrupt actin structural plasticity, rather than global actin conformation. Despite the lower resolution of jordan mutant versus the 
wild-type actomyosin-15 reconstruction, the D-loop is clearly resolved, and the backbone and sidechain of each residue can be fully fit into the density, adopting an "In" conformation indistinguishable from the wild-type reconstruction (Figure 5C,D). Density for the "Out" conformation, on the other hand, is completely absent, demonstrating that the jordan mutant locks the D-loop in the "In" conformation and eliminates its flexibility (Video S4). Thus, the jordan mutation appears to unexpectedly restrict the structural plasticity of the D-loop, presumably through a long-range allosteric effect, rather than eliciting structural rearrangements immediately adjacent to the site of the p.D1647 lesion. While we cannot rule out the possibility that the jordan mutation elicits additional perturbations of myosin-15 or F-actin structure during other steps of the myosin mechanochemical cycle, wild-type myosin-15 most potently stimulates F-actin assembly in the absence of nucleotide, arguing the jordan mutant's defect likely occurs in a strongly-bound state (Moreland, 2021). As restricted D-loop flexibility is the only distinguishable difference between the two rigor structures, we propose that this dysregulation of actin structural plasticity contributes to inhibition of actin assembly by the jordan mutant (Moreland, 2021). Our structures furthermore support a key role for actin residue G48 in conferring D-loop flexibility mediating F-actin assembly. In $\gamma$-actin, the p.G48R substitution (Figure S3B) has been documented to cause progressive hearing loss in human patients (Miyagawa et al., 2015), phenocopying the jordan mutation. While D-loop flexibility at this position is likely a general feature of mammalian actin isoforms (Graceffa and Dominguez, 2003; Otterbein et al., 2001; von der Ecken et al., 2015), our data suggest that it is specifically required for efficient actin polymerization in stereocilia.

\section{The Mg-ADP state of myosin-15 stabilizes the D-loop to restrict actin nucleation}

The ability of myosin- 15 to nucleate F-actin is potently reduced by the presence of ATP

(Moreland, 2021), leading us to hypothesize that other states in the motor's mechanochemical cycle could have distinct effects on actin polymerization by differentially regulating F-actin conformation, as we have previously reported for myosin-6 (Gurel et al., 2017). We next undertook a detailed comparison of the rigor and Mg-ADP-bound actomyosin-15 wild-type structures. The overall conformation of the myosin- 
15 motor domain is highly similar in both reconstructions, with a global RMSD of $0.462 \AA$ for 493

aligned $\mathrm{C}_{\alpha}$ atoms (Figure 6A), indicating an absence of major subdomain rearrangements during ADP release (Figure S7A). Clear density corresponding to ADP is present in the Mg-ADP state cryo-EM map (Figure 6A), arguing that the observed lack of rearrangements is not due to insufficient nucleotide binding by the motor. Both myosin-1 and myosin-6 feature a minor lever-arm swing accompanying ADP release, which has been postulated by us and others to mediate the known force-sensitivity of this step in their mechanochemical cycles (Gurel et al., 2017; Mentes et al., 2018; Nyitrai and Geeves, 2004). As the myosin-15 lever arm does not undergo a substantial transition (Figures 6A, S7A), we speculate that ADP release by myosin-15 is unlikely to be strongly mechanically-gated.

We next compared the conformation of F-actin between the rigor and Mg-ADP structures. Consistent with the lack of conformational rearrangements in the motor domain, ADP release by myosin15 evokes minimal global conformational changes in F-actin (Video S5); correspondingly, the helical rise of the two reconstructions is nearly identical (Figure S1C). Density for the D-loop is clearly resolved in the "In" conformation, positioned as we observed in both the wild-type and jordan mutant rigor reconstructions. However, like the jordan mutant actomyosin-15 reconstruction, density for the "Out" conformation is completely absent (Figure 6B-C, Video S6). As rigor jordan myosin-15 suppresses both D-loop dynamics and actin polymerization, we hypothesized ADP binding would correspondingly reduce the actin-polymerization stimulating activity of wild-type myosin-15 by also stabilizing the D-loop in the "In" conformation.

Consistent with this prediction, we observe a slower rate of actin assembly when saturating $\mathrm{Mg}$ ADP is included along with myosin-15 in pyrene actin polymerization assays versus nucleotide-free conditions (Figure 6D), with an approximate doubling of the half-time to reach steady-state (Figure 6E). Inclusion of Mg-ADP did not substantially affect the assembly rate of actin alone (Figure 6D), suggesting ADP downregulates the polymerization-stimulating activity of myosin-15 rather than perturbing F-actin formation. We next sought to determine if ADP binding by myosin-15 impacted the nucleation or elongation phases of actin polymerization by directly visualizing filament dynamics with Total Internal 
Reflection Fluorescence (TIRF) microscopy (Figure 6F). Relative to actin alone, we observed a striking increase in the density of filaments per unit area in the presence of myosin-15, which was blunted by the inclusion of saturating Mg-ADP (Figure 6F,G), suggesting a specific effect on actin nucleation. When the growth of individual filament plus ends was monitored (Figure 6H), both nucleotide-free and Mg-ADP myosin-15 significantly slowed elongation to $\sim 7.5 \mathrm{~nm} / \mathrm{s}$, relative to $\sim 16 \mathrm{~nm} / \mathrm{s}$ for actin alone (Figure 6I). This rate was indistinguishable from that observed in the presence of the rigor jordan mutant in our companion study (Moreland, 2021). As a further control, the addition of ADP only modestly affected the elongation rate of actin alone (Figure 6I). Myosin-15's opposing effects of stimulating nucleation while reducing elongation strongly suggest that the overall increased F-actin assembly rate is dominated by myosin-15's effect on stimulating nucleation, as previously reported for muscle myosin S1 (Fievez et al., 1997; Lheureux et al., 1993), in a manner which is specifically regulated by myosin-15 nucleotide state.

\section{Discussion:}

Here, we establish structural mechanisms underpinning myosin-15's function in constructing the actin core of stereocilia, a process that is critical for hearing. Our actomyosin-15 cryo-EM structures, the first myosin-15 motor domain structures, to our knowledge, of any kind, assign the likely mechanistic deficiencies associated with many DFNB3 deafness mutations, as well as provide a framework for interpreting novel clinical variants (Figure 2). Many mutations are intuitively anticipated to impact the motor's mechanochemical cycle. However, our structure also highlights surface substitutions which are not readily interpreted through this lens, suggesting additional functions of the myosin-15 motor domain important for hearing remain to be identified, such as serving as a protein-protein interaction hub. Our studies additionally reveal how myosin-15 modulates actin's structural landscape to enhance F-actin nucleation in a manner controlled by the motor's nucleotide state. We speculate this activity could be harnessed to provide fine-tuned control of F-actin assembly at stereocilia tips, facilitating the precise control of stereocilia height required for sound detection. 
Our cryo-EM reconstructions and associated functional data reveal subtle structural plasticity in the actin D-loop which mediates regulated F-actin assembly by myosin-15. While substantial D-loop rearrangements accompanying the soluble G-actin to polymerized F-actin conformational transition are well-established (Dominguez and Holmes, 2011), the extent and functional role of D-loop rearrangements within F-actin remain controversial. Substantially different "open" and "closed" conformations have been reported in cryo-EM reconstructions of F-actin in the presence of stabilizing drugs and actin nucleotidestate analogs (Merino et al., 2018; Pospich et al., 2020) leading to speculation that an open-to-closed transition is associated with nucleotide hydrolysis and phosphate release concomitant with F-actin polymerization. However, others have reported minimal rearrangements when comparing highly similar conditions, with the D-loop constitutively adopting a closed conformation in F-actin (Chou and Pollard, 2019; Das et al., 2020). The data we present here reveal the closed D-loop conformation in ADP F-actin to be a mixture of "In" and "Out" states whose occupancy can be manipulated by myosin-15 engagement. Wild-type rigor myosin-15 binding retains both the "In" and "Out" D-loop conformations and strongly stimulates actin polymerization, while ADP-bound wild-type myosin-15 and rigor jordan mutant-15 both lock the D-loop in the "In" state, with corresponding moderate and striking reductions, respectively, in Factin-polymerization stimulation activity (Figure 7). Collectively, these data suggest that the co-existence of the "In" and "Out" D-loop conformations promotes polymerization relative to locking the D-loop in the "In" conformation, a model which is consistent with a recent report demonstrating that constraining D-loop flexibility with short-distance crosslinkers was refractory to actin polymerization and stimulated F-actin disassembly (Das et al., 2020). Previous studies have further shown that muscle myosin S1 can rescue polymerization of actin featuring a proteolytically disrupted D-loop (Wawro et al., 2005), consistent with a role for myosin in modulating D-loop dynamics during actin polymerization.

Nevertheless, additional mechanisms are likely required to explain wild-type myosin-15's stimulation of actin polymerization and its striking disruption by the jordan deafness mutation. While actin polymerization by muscle myosin $\mathrm{S} 1$ has been subjected to extensive biochemical scrutiny, the exact mechanism remains controversial. Chaussepied and colleagues have suggested that each S1 engages 
a single G-actin, which then stimulates polymerization through a standard nucleation-elongation mechanism (Lheureux et al., 1993). Carlier and co-workers, on the other hand, have suggested that each S1 engages two G-actins, forming trimers with an F-actin-like actin-actin longitudinal bond which coalesce into filaments through a mechanism distinct from standard polymerization (Fievez et al., 1997). While our data do not directly discriminate between these mechanisms, our observation that the jordan mutation is localized to the HLH interface, which bridges the contact between two longitudinally-adjacent protomers, supports a model in which high-affinity myosin-15 binding promotes longitudinal interactions to stimulate polymerization (Figure 7). The modestly lower affinity of ADP-myosin 15 and strikingly lower affinity of the jordan mutant for F-actin, relative to the rigor wild-type (Moreland, 2021), also likely makes a major contribution to determining their potency in stimulating F-actin formation. This defect occurring at the level of nuclei formation, rather than elongation, is consistent with our observation that F-actin elongation rates are indistinguishable in the presence of ADP-bound or rigor wild-type myosin-15 (Figure 6H and I), as well as the rigor jordan mutant (Moreland, 2021), all of which slow actin polymerization relative to G-actin alone. Regardless, diminished affinity of the jordan mutant cannot be the sole explanation for its polymerization defect, as the presence of this myosin, which nevertheless binds F-actin with measurable affinity in an almost identical pose to the wild-type, actually initially suppresses the rate of F-actin formation relative to G-actin alone (Moreland, 2021). The most parsimonious explanation for this effect is through the suppression of D-loop flexibility as highlighted above, emphasizing the contributions of both myosin- 15 bridging actin monomers to stimulate nucleus formation, and the maintenance of actin conformational plasticity to the accelerated actin polymerization we observe (Figure 7).

Our studies define a biophysical and structural framework for tunable stimulation of actin polymerization by myosin- 15 which could be modulated by the local biochemical environment at the tips of stereocilia, the major sites of actin polymerization. We speculate that hair cells harness the differential efficiency of actin nucleation by myosin-15 in distinct nucleotide states as a regulatable mechanistic component of stereocilia height control. It is feasible that the ADP-bound state, which substantially 
accelerates actin polymerization, albeit less than the rigor state, is sufficient for supporting myosin-15's contribution to actin polymerization in the tip compartment. Within this framework, control over myosin15's actin nucleation could be exerted by the availability of ATP versus ADP (Moreland, 2021), which has previously been reported to be dynamically maintained by creatine kinase in stereocilia during mechanotransduction and adaptation (Shin et al., 2007) in a manner which may vary in different species and hair cell subtypes (Krey and Barr-Gillespie, 2019). Our structural data furthermore suggest that ADP release is unlikely to be mechanically-gated in myosin- 15 , which could facilitate the motor populating a nucleotide-free state in the cell in order to boost its nucleation activity in certain ATP / ADP concentration regimes. In future studies, it will be important to determine the dynamic availability of ATP and ADP in the tip compartment of developing stereocilia, and how myosin-15's direct effects on actin polymerization are coordinated with the WHRN-EPS8-GPSM2-GNAI3 elongation network that it transports. Other environmental conditions known to impact stereocilium integrity, such as oxidative stress (Wagner and Shin, 2019), could also potentially impact stimulated actin polymerization by myosin15 , both by dysregulation of nucleotide availability and by direct modification of the myosin-15 protein, disrupting the actin-myosin interface in a manner conceptually analogous to the jordan mutation. Finally, our observation that the jordan mutation occurs at a conserved position suggests that other unconventional myosins could also feasibly stimulate actin polymerization in stereocilia, as well as in diverse physiological contexts beyond hair cells. The mechanistic framework we establish here will broadly facilitate investigating the functional role of unconventional myosins in actin assembly dynamics.

\section{Acknowledgements:}

We gratefully acknowledge Yasuharu Takagi and James Sellers (NHLBI DIR) for assistance with myosin biochemistry, Rabia Faridi (NIDCD DIR) for a curated list of DFNB3 mutations, and Peter BarrGillespie for critical feedback. We also thank Johanna Sotiris and Mark Ebrahim from the Rockefeller University Cryo-EM Resource Center (CEMRC) for their assistance with data collection. R.G. was supported by an H. Li Memorial Fellowship and P.G. by a Rockefeller Women in Science Fellowship. 
This research was supported (in part) by the Intramural Research Program of the NIH, NIDCD DC000039

to Thomas B. Friedman. The work was additionally funded by grants from the Medical Research Council

to M.R.B. (MC_UP_1503/2), the Pew Charitable Trusts and Irma T. Hirschl / Monique Weill-Caulier

Trust to G.M.A., as well as National Institutes of Health grants to J.E.B (R01DC018827) and G.M.A.

(DP5OD017885 and R01GM141044). The content of this manuscript is solely the responsibility of the authors and does not necessarily represent the official views of the National Institutes of Health.

\section{$\underline{\text { Author Contributions: }}$}

R.G.: Conceptualization, Methodology, Investigation, Formal analysis, Visualization, Writing -

Original draft. F.J.: Investigation, Formal analysis. Z.G.M.: Investigation, Formal analysis. M.J.R.:

Methodology, Formal analysis. S.E.R.: Investigation, Formal analysis. P.G. Methodology, Investigation.

A.S.: Methodology, Investigation. M.R.B.: Resources. J.E.B.: Conceptualization, Visualization, Project administration, Funding acquisition, Writing - Original draft, Supervision: G.M.A. Conceptualization, Formal analysis, Visualization, Project administration, Funding acquisition, Writing - Original draft, Supervision. All authors: Writing - Review \& editing.

\section{Data Availability Statement:}

Cryo-EM maps and corresponding atomic models have been deposited in the EMDB and PDB databases. Accession codes are as follows: rigor wild-type myosin-15 bound F-actin: EMDB: EMD24322, PDB: 7R91; rigor jordan myosin-15 bound F-actin: EMDB: EMD-24400, PDB: 7RB9; ADP wild-type myosin-15 bound F-actin: EMDB: EMD-24399, PDB: 7RB8; F-actin alone: EMDB: EMD24321, PDB: 7R8V. 


\section{Reference:}

Anderson, D.W., Probst, F.J., Belyantseva, I.A., Fridell, R.A., Beyer, L., Martin, D.M., Wu, D., Kachar, B., Friedman, T.B., Raphael, Y., et al. (2000). The motor and tail regions of myosin XV are critical for normal structure and function of auditory and vestibular hair cells. Hum Mol Genet 9, 1729-1738.

Barr-Gillespie, P.G. (2015). Assembly of hair bundles, an amazing problem for cell biology. Mol Biol Cell 26, 2727-2732.

Belyantseva, I.A., Boger, E.T., and Friedman, T.B. (2003). Myosin XVa localizes to the tips of inner ear sensory cell stereocilia and is essential for staircase formation of the hair bundle. Proc Natl Acad Sci U S A $100,13958-13963$.

Belyantseva, I.A., Boger, E.T., Naz, S., Frolenkov, G.I., Sellers, J.R., Ahmed, Z.M., Griffith, A.J., and Friedman, T.B. (2005). Myosin-XVa is required for tip localization of whirlin and differential elongation of hair-cell stereocilia. Nat Cell Biol 7, 148-156.

Bird, J.E., Takagi, Y., Billington, N., Strub, M.P., Sellers, J.R., and Friedman, T.B. (2014). Chaperoneenhanced purification of unconventional myosin 15, a molecular motor specialized for stereocilia protein trafficking. Proc Natl Acad Sci U S A 111, 12390-12395.

Chou, S.Z., and Pollard, T.D. (2019). Mechanism of actin polymerization revealed by cryo-EM structures of actin filaments with three different bound nucleotides. Proc Natl Acad Sci U S A 116, 4265-4274.

Crevenna, A.H., Arciniega, M., Dupont, A., Mizuno, N., Kowalska, K., Lange, O.F., Wedlich-Soldner, R., and Lamb, D.C. (2015). Side-binding proteins modulate actin filament dynamics. Elife 4.

Das, S., Ge, P., Oztug Durer, Z.A., Grintsevich, E.E., Zhou, Z.H., and Reisler, E. (2020). D-loop Dynamics and Near-Atomic-Resolution Cryo-EM Structure of Phalloidin-Bound F-Actin. Structure 28, 586-593 e583.

Delprat, B., Michel, V., Goodyear, R., Yamasaki, Y., Michalski, N., El-Amraoui, A., Perfettini, I., Legrain, P., Richardson, G., Hardelin, J.P., et al. (2005). Myosin XVa and whirlin, two deafness gene products required for hair bundle growth, are located at the stereocilia tips and interact directly. Hum Mol Genet 14, 401-410.

DeRosier, D.J., Tilney, L.G., and Egelman, E. (1980). Actin in the inner ear: the remarkable structure of the stereocilium. Nature 287, 291-296.

Disanza, A., Carlier, M.F., Stradal, T.E., Didry, D., Frittoli, E., Confalonieri, S., Croce, A., Wehland, J., Di Fiore, P.P., and Scita, G. (2004). Eps8 controls actin-based motility by capping the barbed ends of actin filaments. Nat Cell Biol 6, 1180-1188.

Dominguez, R., and Holmes, K.C. (2011). Actin structure and function. Annu Rev Biophys 40, 169-186.

Drummond, M.C., Barzik, M., Bird, J.E., Zhang, D.S., Lechene, C.P., Corey, D.P., Cunningham, L.L., and Friedman, T.B. (2015). Live-cell imaging of actin dynamics reveals mechanisms of stereocilia length regulation in the inner ear. Nat Commun 6, 6873.

Drummond, M.C., Belyantseva, I.A., Friderici, K.H., and Friedman, T.B. (2012). Actin in hair cells and hearing loss. Hear Res 288, 89-99. 
Durer, Z.A., Kudryashov, D.S., Sawaya, M.R., Altenbach, C., Hubbell, W., and Reisler, E. (2012). Structural states and dynamics of the D-loop in actin. Biophys J 103, 930-939.

Egelman, E.H. (2007). The iterative helical real space reconstruction method: surmounting the problems posed by real polymers. J Struct Biol 157, 83-94.

Fang, Q., Indzhykulian, A.A., Mustapha, M., Riordan, G.P., Dolan, D.F., Friedman, T.B., Belyantseva, I.A., Frolenkov, G.I., Camper, S.A., and Bird, J.E. (2015). The 133-kDa N-terminal domain enables myosin 15 to maintain mechanotransducing stereocilia and is essential for hearing. Elife 4.

Fievez, S., Carlier, M.F., and Pantaloni, D. (1997). Mechanism of myosin subfragment-1-induced assembly of CaG-actin and MgG-actin into F-actin-S1-decorated filaments. Biochemistry 36, 1184311850 .

Furch, M., Remmel, B., Geeves, M.A., and Manstein, D.J. (2000). Stabilization of the actomyosin complex by negative charges on myosin. Biochemistry 39, 11602-11608.

Galkin, V.E., Orlova, A., Schroder, G.F., and Egelman, E.H. (2010). Structural polymorphism in F-actin. Nat Struct Mol Biol 17, 1318-1323.

Graceffa, P., and Dominguez, R. (2003). Crystal structure of monomeric actin in the ATP state. Structural basis of nucleotide-dependent actin dynamics. J Biol Chem 278, 34172-34180.

Gurel, P.S., Kim, L.Y., Ruijgrok, P.V., Omabegho, T., Bryant, Z., and Alushin, G.M. (2017). Cryo-EM structures reveal specialization at the myosin VI-actin interface and a mechanism of force sensitivity. Elife 6.

Hertzog, M., Milanesi, F., Hazelwood, L., Disanza, A., Liu, H., Perlade, E., Malabarba, M.G., Pasqualato, S., Maiolica, A., Confalonieri, S., et al. (2010). Molecular basis for the dual function of Eps8 on actin dynamics: bundling and capping. PLoS Biol 8, e1000387.

Houdusse, A., and Sweeney, H.L. (2016). How Myosin Generates Force on Actin Filaments. Trends Biochem Sci 41, 989-997.

Krey, J.F., and Barr-Gillespie, P.G. (2019). Molecular Composition of Vestibular Hair Bundles. Cold Spring Harb Perspect Med 9.

Lheureux, K., Forne, T., and Chaussepied, P. (1993). Interaction and polymerization of the G-actinmyosin head complex: effect of DNase I. Biochemistry 32, 10005-10014.

Manor, U., Disanza, A., Grati, M., Andrade, L., Lin, H., Di Fiore, P.P., Scita, G., and Kachar, B. (2011). Regulation of stereocilia length by myosin XVa and whirlin depends on the actin-regulatory protein Eps8. Curr Biol 21, 167-172.

Mauriac, S.A., Hien, Y.E., Bird, J.E., Carvalho, S.D., Peyroutou, R., Lee, S.C., Moreau, M.M., Blanc, J.M., Geyser, A., Medina, C., et al. (2017). Defective Gpsm2/Galphai3 signalling disrupts stereocilia development and growth cone actin dynamics in Chudley-McCullough syndrome. Nat Commun 8, 14907.

Mburu, P., Mustapha, M., Varela, A., Weil, D., El-Amraoui, A., Holme, R.H., Rump, A., Hardisty, R.E., Blanchard, S., Coimbra, R.S., et al. (2003). Defects in whirlin, a PDZ domain molecule involved in 
stereocilia elongation, cause deafness in the whirler mouse and families with DFNB31. Nat Genet 34, 421-428.

Mentes, A., Huehn, A., Liu, X., Zwolak, A., Dominguez, R., Shuman, H., Ostap, E.M., and Sindelar, C.V. (2018). High-resolution cryo-EM structures of actin-bound myosin states reveal the mechanism of myosin force sensing. Proc Natl Acad Sci U S A 115, 1292-1297.

Merino, F., Pospich, S., Funk, J., Wagner, T., Kullmer, F., Arndt, H.D., Bieling, P., and Raunser, S. (2018). Structural transitions of F-actin upon ATP hydrolysis at near-atomic resolution revealed by cryoEM. Nat Struct Mol Biol 25, 528-537.

Miller, L., Phillips, M., and Reisler, E. (1988). Polymerization of G-actin by myosin subfragment 1. J Biol Chem 263, 1996-2002.

Miyagawa, M., Nishio, S.Y., Ichinose, A., Iwasaki, S., Murata, T., Kitajiri, S., and Usami, S. (2015). Mutational spectrum and clinical features of patients with ACTG1 mutations identified by massively parallel DNA sequencing. Ann Otol Rhinol Laryngol 124 Suppl 1, 84S-93S.

Moreland, Z.G., Jiang, F., Aguilar, C., Barzik, M., Gong, R., Shams, A., Faaborg-Andersen, C., Werth, J.C., Harley, R., Sutton, D.C., Cole, S.M., Parker, A., Morse, S., Wilson, E., Takagi, Y., Sellers, J.R., Brown, S.D.M., Friedman, T.B., Alushin, G.M., Bowl M.R., Bird, J.E. (2021). Myosin-driven Nucleation of Actin Filaments Drives Stereocilia Development Critical for Hearing. BioRxiv 2021.07.09.451618.

Murphy, C.T., and Spudich, J.A. (1999). The sequence of the myosin 50-20K loop affects Myosin's affinity for actin throughout the actin-myosin ATPase cycle and its maximum ATPase activity. Biochemistry 38, 3785-3792.

Narayanan, P., Chatterton, P., Ikeda, A., Ikeda, S., Corey, D.P., Ervasti, J.M., and Perrin, B.J. (2015). Length regulation of mechanosensitive stereocilia depends on very slow actin dynamics and filamentsevering proteins. Nat Commun 6, 6855.

Nyitrai, M., and Geeves, M.A. (2004). Adenosine diphosphate and strain sensitivity in myosin motors. Philos Trans R Soc Lond B Biol Sci 359, 1867-1877.

Onishi, H., Mikhailenko, S.V., and Morales, M.F. (2006). Toward understanding actin activation of myosin ATPase: the role of myosin surface loops. Proc Natl Acad Sci U S A 103, 6136-6141.

Otterbein, L.R., Graceffa, P., and Dominguez, R. (2001). The crystal structure of uncomplexed actin in the ADP state. Science 293, 708-711.

Pollard, T.D. (2016). Actin and Actin-Binding Proteins. Cold Spring Harb Perspect Biol 8. Pospich, S., Merino, F., and Raunser, S. (2020). Structural Effects and Functional Implications of Phalloidin and Jasplakinolide Binding to Actin Filaments. Structure 28, 437-449 e435.

Probst, F.J., Fridell, R.A., Raphael, Y., Saunders, T.L., Wang, A., Liang, Y., Morell, R.J., Touchman, J.W., Lyons, R.H., Noben-Trauth, K., et al. (1998). Correction of deafness in shaker-2 mice by an unconventional myosin in a BAC transgene. Science 280, 1444-1447.

Rehman, A.U., Bird, J.E., Faridi, R., Shahzad, M., Shah, S., Lee, K., Khan, S.N., Imtiaz, A., Ahmed, Z.M., Riazuddin, S., et al. (2016). Mutational Spectrum of MYO15A and the Molecular Mechanisms of DFNB3 Human Deafness. Hum Mutat 37, 991-1003. 
Risi, C., Schafer, L.U., Belknap, B., Pepper, I., White, H.D., Schroder, G.F., and Galkin, V.E. (2021). High-Resolution Cryo-EM Structure of the Cardiac Actomyosin Complex. Structure 29, 50-60 e54.

Rzadzinska, A.K., Schneider, M.E., Davies, C., Riordan, G.P., and Kachar, B. (2004). An actin molecular treadmill and myosins maintain stereocilia functional architecture and self-renewal. J Cell Biol 164, 887897.

Shin, J.B., Streijger, F., Beynon, A., Peters, T., Gadzala, L., McMillen, D., Bystrom, C., Van der Zee, C.E., Wallimann, T., and Gillespie, P.G. (2007). Hair bundles are specialized for ATP delivery via creatine kinase. Neuron 53, 371-386.

Sloan-Heggen, C.M., Bierer, A.O., Shearer, A.E., Kolbe, D.L., Nishimura, C.J., Frees, K.L., Ephraim, S.S., Shibata, S.B., Booth, K.T., Campbell, C.A., et al. (2016). Comprehensive genetic testing in the clinical evaluation of 1119 patients with hearing loss. Hum Genet 135, 441-450.

Tadenev, A.L.D., Akturk, A., Devanney, N., Mathur, P.D., Clark, A.M., Yang, J., and Tarchini, B. (2019). GPSM2-GNAI Specifies the Tallest Stereocilia and Defines Hair Bundle Row Identity. Curr Biol 29, 921-934 e924.

Tarchini, B., Tadenev, A.L., Devanney, N., and Cayouette, M. (2016). A link between planar polarity and staircase-like bundle architecture in hair cells. Development 143, 3926-3932.

Tilney, L.G., Derosier, D.J., and Mulroy, M.J. (1980). The organization of actin filaments in the stereocilia of cochlear hair cells. J Cell Biol 86, 244-259.

Tilney, L.G., Tilney, M.S., and DeRosier, D.J. (1992). Actin filaments, stereocilia, and hair cells: how cells count and measure. Annu Rev Cell Biol 8, 257-274.

Uyeda, T.Q., Ruppel, K.M., and Spudich, J.A. (1994). Enzymatic activities correlate with chimaeric substitutions at the actin-binding face of myosin. Nature 368, 567-569.

Varkuti, B.H., Yang, Z., Kintses, B., Erdelyi, P., Bardos-Nagy, I., Kovacs, A.L., Hari, P., Kellermayer, M., Vellai, T., and Malnasi-Csizmadia, A. (2012). A novel actin binding site of myosin required for effective muscle contraction. Nat Struct Mol Biol 19, 299-306.

Varkuti, B.H., Yang, Z., and Malnasi-Csizmadia, A. (2015). Structural model of weak binding actomyosin in the prepowerstroke state. J Biol Chem 290, 1679-1688.

von der Ecken, J., Heissler, S.M., Pathan-Chhatbar, S., Manstein, D.J., and Raunser, S. (2016). Cryo-EM structure of a human cytoplasmic actomyosin complex at near-atomic resolution. Nature 534, 724-728.

von der Ecken, J., Muller, M., Lehman, W., Manstein, D.J., Penczek, P.A., and Raunser, S. (2015). Structure of the F-actin-tropomyosin complex. Nature 519, 114-117.

Wagner, E.L., and Shin, J.B. (2019). Mechanisms of Hair Cell Damage and Repair. Trends Neurosci 42, 414-424.

Wang, A., Liang, Y., Fridell, R.A., Probst, F.J., Wilcox, E.R., Touchman, J.W., Morton, C.C., Morell, R.J., Noben-Trauth, K., Camper, S.A., et al. (1998). Association of unconventional myosin MYO15 mutations with human nonsyndromic deafness DFNB3. Science 280, 1447-1451. 
Wawro, B., Khaitlina, S.Y., Galinska-Rakoczy, A., and Strzelecka-Golaszewska, H. (2005). Role of actin DNase-I-binding loop in myosin subfragment 1-induced polymerization of G-actin: implications for the mechanism of polymerization. Biophys J 88, 2883-2896.

Zampini, V., Ruttiger, L., Johnson, S.L., Franz, C., Furness, D.N., Waldhaus, J., Xiong, H., Hackney, C.M., Holley, M.C., Offenhauser, N., et al. (2011). Eps8 regulates hair bundle length and functional maturation of mammalian auditory hair cells. PLoS Biol 9, e1001048.

Zhang, D.S., Piazza, V., Perrin, B.J., Rzadzinska, A.K., Poczatek, J.C., Wang, M., Prosser, H.M., Ervasti, J.M., Corey, D.P., and Lechene, C.P. (2012). Multi-isotope imaging mass spectrometry reveals slow protein turnover in hair-cell stereocilia. Nature 481, 520-524.

Zhang, F., Xu, L., Xiao, Y., Li, J., Bai, X., and Wang, H. (2018). Three MYO15A Mutations Identified in One Chinese Family with Autosomal Recessive Nonsyndromic Hearing Loss. Neural Plast 2018, 5898025 .

Zhang, J., Guan, J., Wang, H., Yin, L., Wang, D., Zhao, L., Zhou, H., and Wang, Q. (2019). Genotypephenotype correlation analysis of MYO15A variants in autosomal recessive non-syndromic hearing loss. BMC Med Genet 20, 60.

Zivanov, J., Nakane, T., Forsberg, B.O., Kimanius, D., Hagen, W.J., Lindahl, E., and Scheres, S.H. (2018). New tools for automated high-resolution cryo-EM structure determination in RELION-3. Elife 7. 
A

1

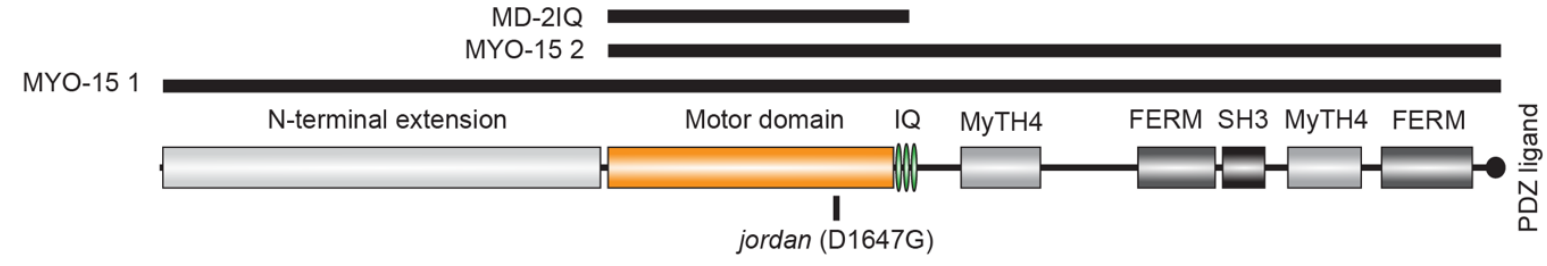

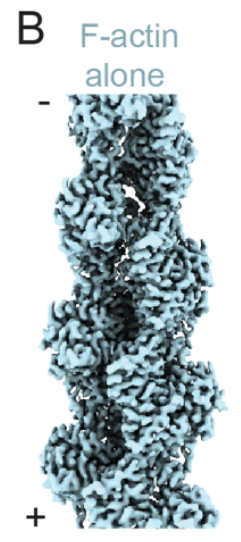

C

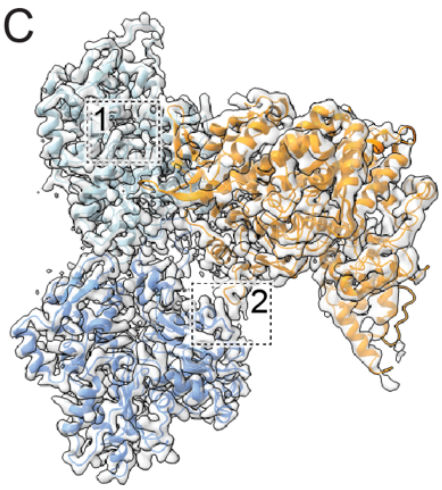

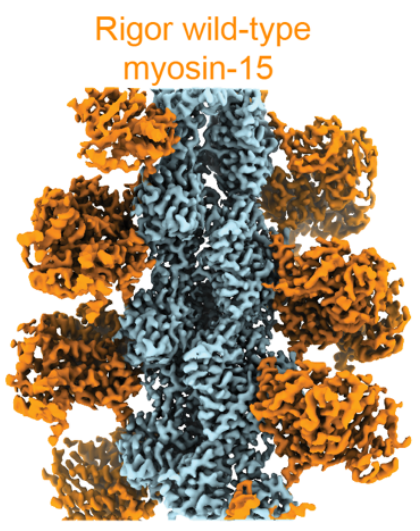

D

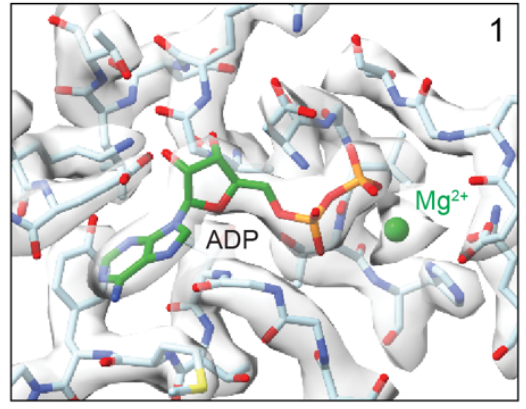

Rigor jordan myosin-15

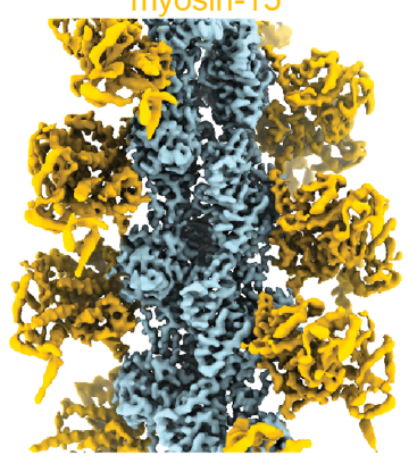

ADP wild-type

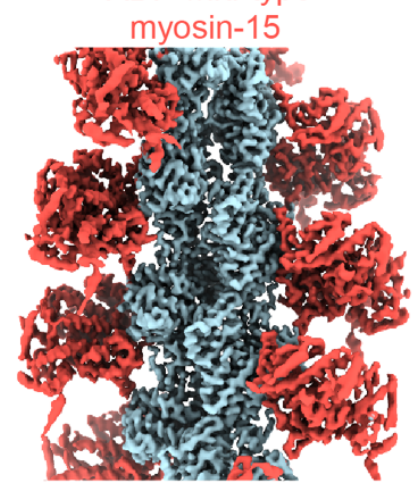

E

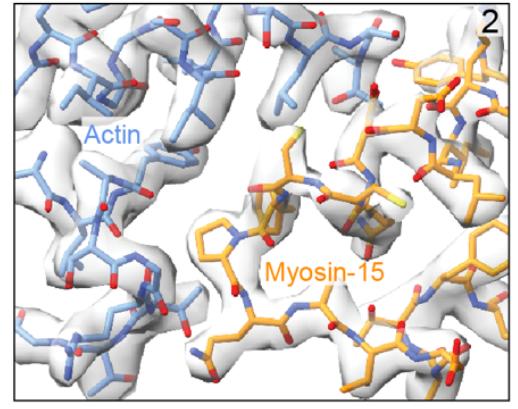

Figure 1. Cryo-EM structures of actomyosin-15 complexes. (A) Diagram of M. musculus myosin-15 primary structure. (B) Cryo-EM maps of F-actin alone and indicated actomyosin-15 complexes. (C) Cryo-EM map and ribbon diagram of two actin and one myosin-15 subunits from the rigor wild-type actomyosin-15 reconstruction. Numbered boxes correspond to detail views in subsequent panels. (D) Cryo-EM map and stick models around actin's ADP binding pocket. (E) Cryo-EM map and stick models at the actin-myosin loop 3 interface. 


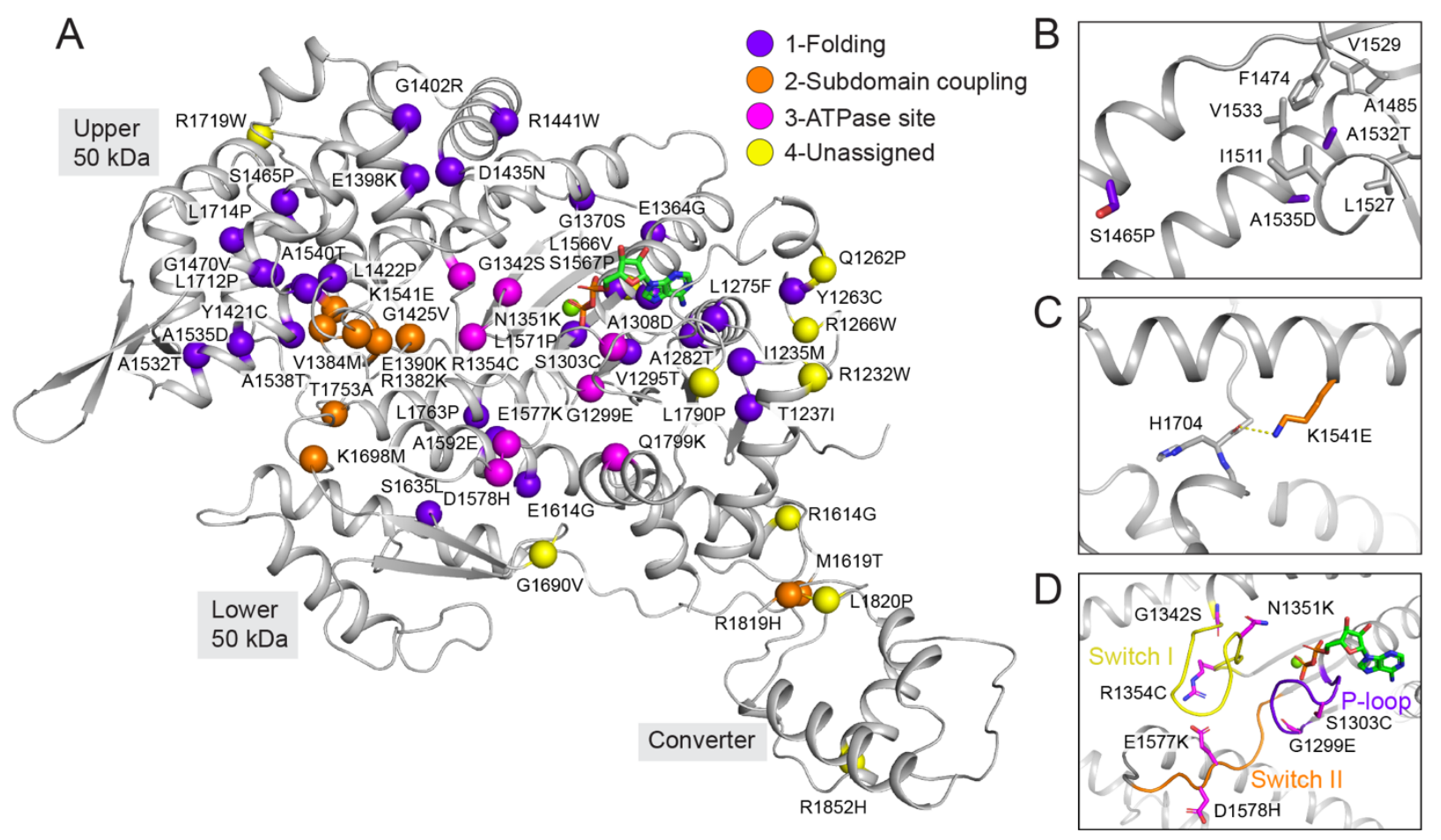

Figure 2. Structural mapping of deafness causing myosin-15 mutations. (A) Ribbon diagram of the myosin-15 motor domain with $\alpha$-carbons at sites of deafness causing mutations shown as spheres, colored according to the indicated mechanistic categories. (B) Detail view of mutations anticipated to disrupt folding. (C) Detail view of mutation anticipated to affect subdomain rearrangements. (D) Detail view of mutations in the switch I, II and P-loop regions anticipated to impact ATPase activity. 

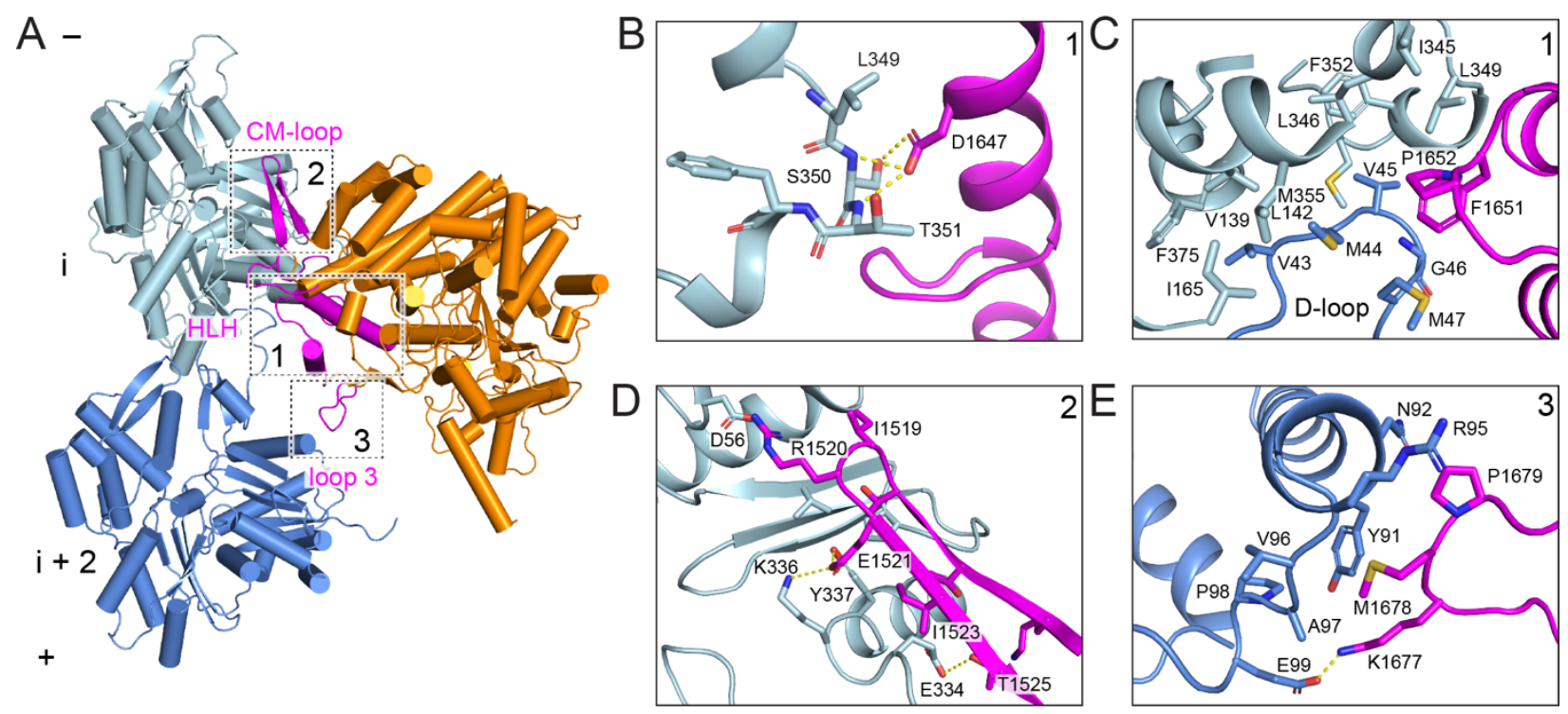

Figure 3. Interactions at the rigor wild-type actomyosin-15 interface. (A) Atomic model of the interface between two actin subunits and rigor wild-type myosin-15. Actin subunits $i, i+2$, and myosin- 15 are colored in light blue, cornflower blue, and dark orange, respectively. The indicated myosin- 15 actin interaction motifs are highlighted in magenta, and numbered boxes correspond to detail views in subsequent panels. (B) Hydrogen bonding interactions between myosin-15 residue D1647 and actin residues S352 and T353. (C) Hydrophobic interactions formed between the D-loop of actin subunit i+2, subdomain 1 and 3 of actin subunit $i$, and the helix-loop-helix (HLH) motif of myosin-15. (D) Interface between the CM loop of myosin-15 and subdomain 3 of actin subunit i. (E) Contacts between loop 3 of myosin-15 and subdomain 1 of actin subunit $i+2$. 
A -

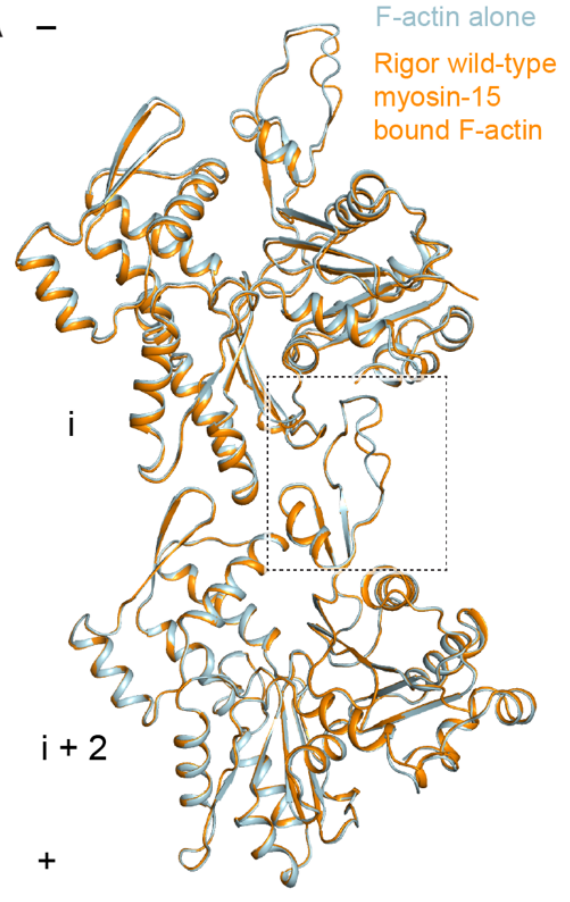

$\mathrm{B}$

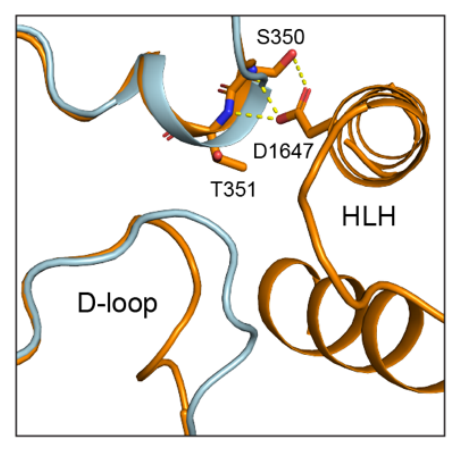

D

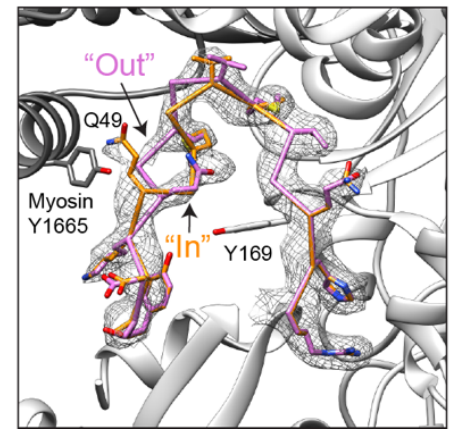

C

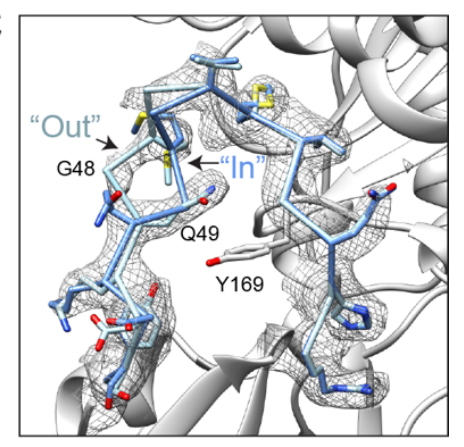

E

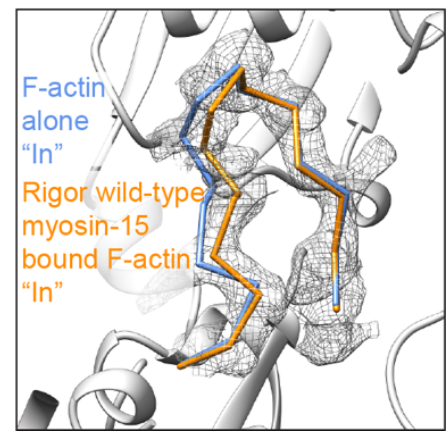

Figure 4. Myosin-15 binding remodels F-actin while maintaining D-loop flexibility. (A) Ribbon

diagram of two actin subunits in the presence and absence of myosin-15, superimposed on subunit i. (B) Structural comparison at the interface formed by two longitudinally adjacent actin subunits and the myosin-15 HLH. Bare F-actin and rigor wild-type actomyosin-15 are colored in light blue and dark orange, respectively. (C) Segmented cryo-EM density of the bare F-actin D-loop rendered at 0.036 RMS, superimposed with stick models of the "Out" and "In" D-loop conformations colored in light blue and cornflower blue, respectively. (D) Segmented cryo-EM density of the rigor wild-type myosin-15 bound actin D-loop, superimposed with stick models of the "Out" and "In" D-loop conformations colored in dark orange and pink, respectively. (E) Backbone representation of the "In" conformation of the indicated models, superimposed on the segmented cryo-EM density the of myosin-15 bound F-actin Dloop. 

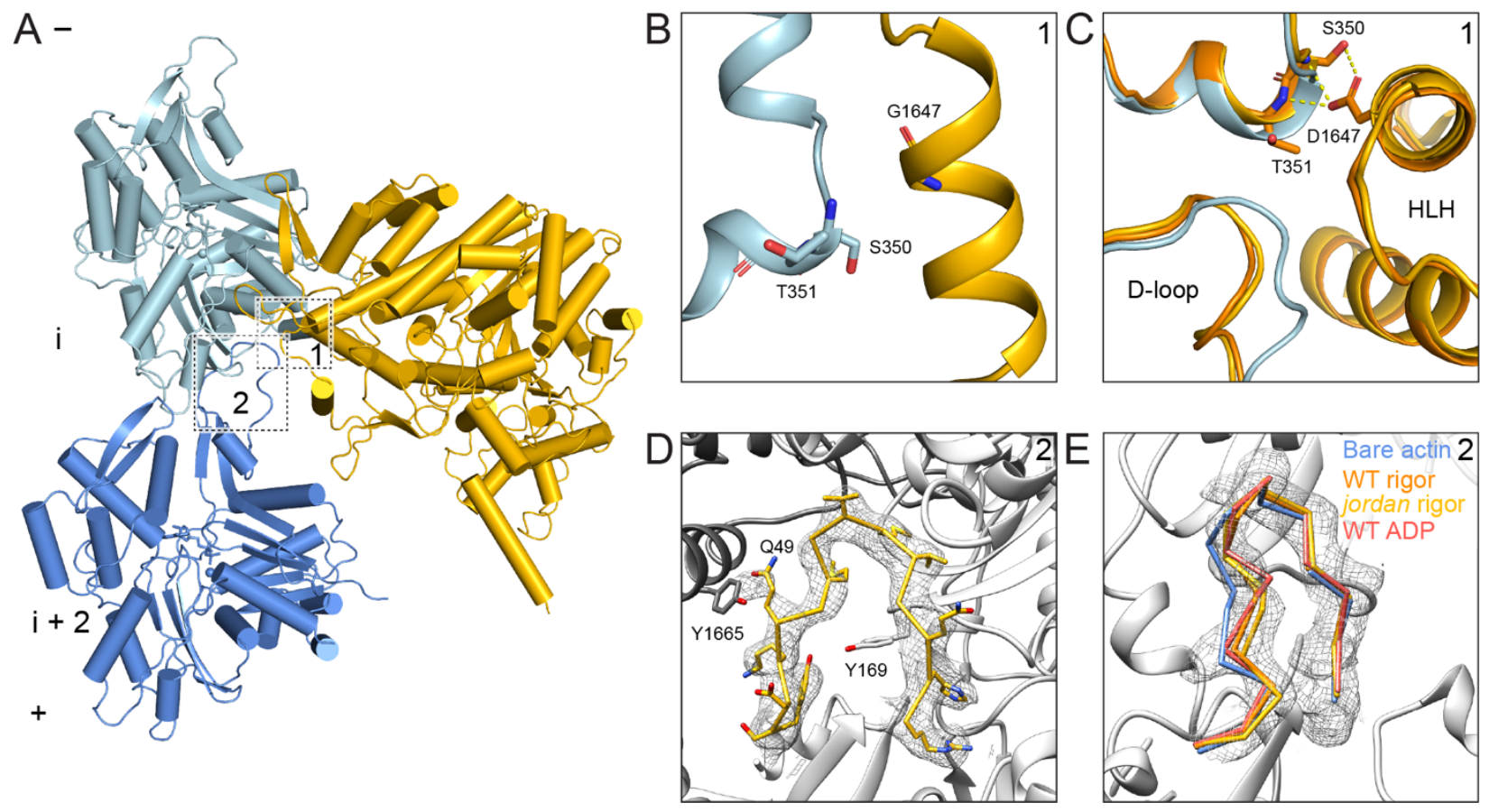

Figure 5. Binding by rigor jordan mutant myosin-15 locks the actin D-loop. (A) Atomic model of the interface between two actin subunits and rigor jordan myosin-15. Actin subunits i, $\mathrm{i}+2$, and myosin-15 are colored in light blue, cornflower blue, and light orange, respectively. Numbered boxes correspond to detail views in subsequent panels. (B) Actomyosin interface at the site of the p.D1647G mutation. (C) Structural comparison of bare F-actin (light blue), rigor wild-type (dark orange), and jordan mutant (gold) myosin-15 bound F-actin at the interface formed by two longitudinally adjacent actin subunits and the myosin HLH. (D) Segmented cryo-EM density and atomic model of the rigor jordan mutant actomyosin15 D-loop. (E) Backbone diagram of the three "In" D-loop conformation models superimposed on segmented cryo-EM density of the rigor jordan mutant actomyosin-15 D-loop. 
A

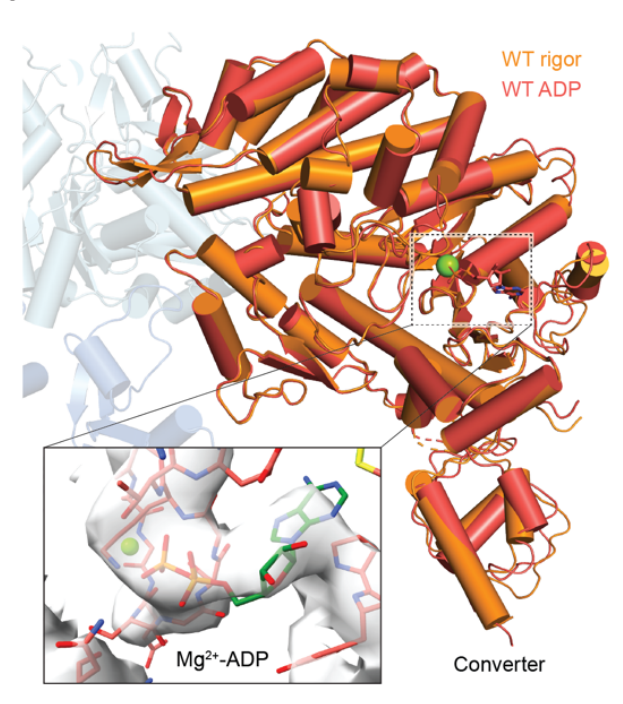

D

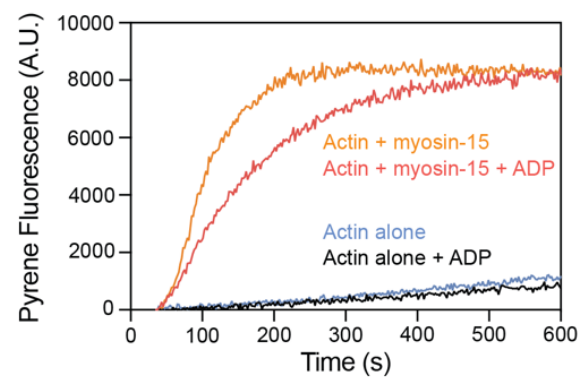

$\mathrm{F}$

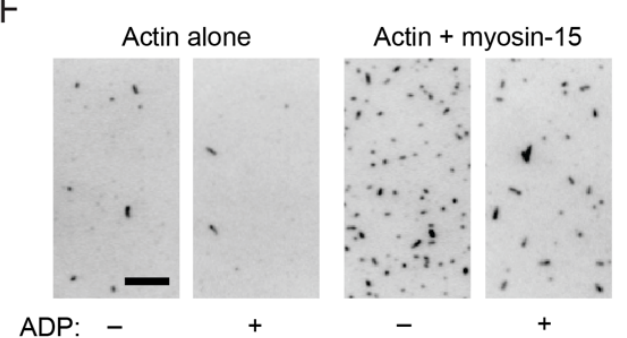

G

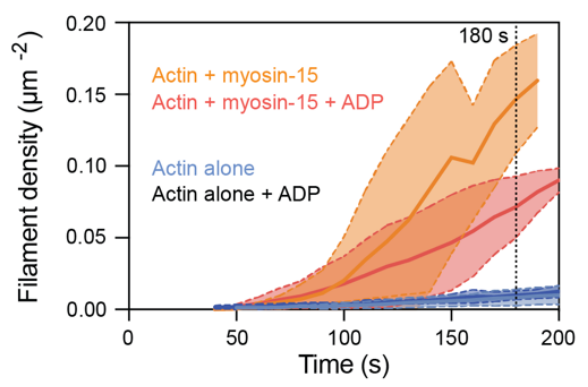

B

C
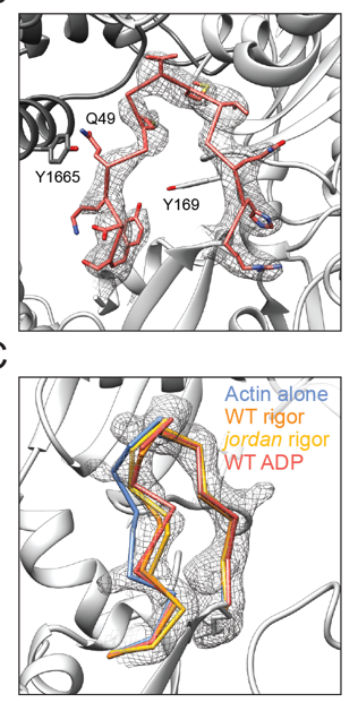

E
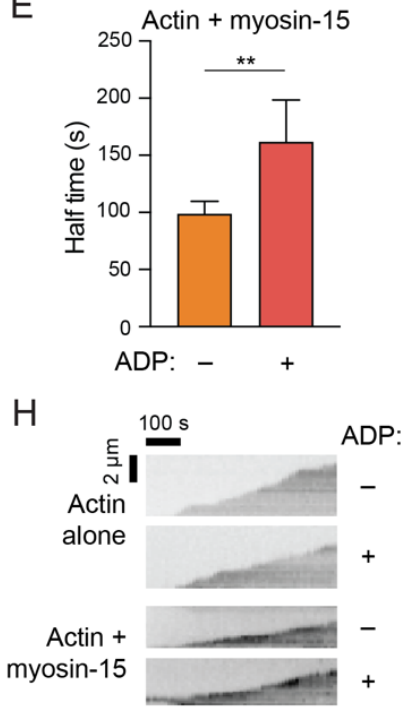

।

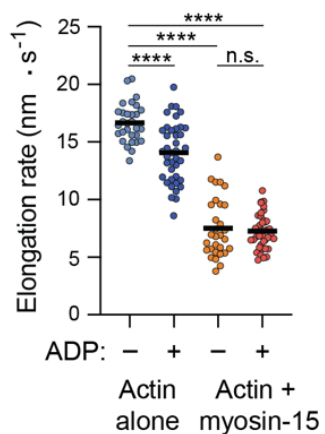

Figure 6. ADP bound myosin-15 locks the D-loop, blunting F-actin stimulation. (A) Superposition of rigor and ADP wild-type actomyosin-15 atomic models. Actins from the rigor atomic model are shown in shades of transparent blue. Inset: Cryo-EM density in the nucleotide-binding pocket of ADP wild-type myosin-15. (B) Cryo-EM map and corresponding atomic model of actin's D-loop in ADP wild-type actomyosin-15. (C) Backbone positioning of "In" D-loop conformation in the indicated models, superimposed on the segmented cryo-EM map of the ADP wild-type actomyosin-15 D-loop. 
bioRxiv preprint doi: https://doi.org/10.1101/2021.07.09.451843; this version posted July 12, 2021. The copyright holder for this preprint (which

was not certified by peer review) is the author/funder, who has granted bioRxiv a license to display the preprint in perpetuity. It is made available under aCC-BY-NC-ND 4.0 International license.

(D) Representative pyrene actin polymerization assays in the presence and absence of myosin-15 and ADP. Actin, $2 \mu \mathrm{M}$; myosin-15, $1 \mu \mathrm{M}$; Mg-ADP, $100 \mu \mathrm{M}$. (E) Quantification of the time to half-maximal pyrene signal saturation in the presence of myosin-15 with or without ADP. Error bars represent S.D. $\mathrm{N}=$ 5 from 3 independent protein purifications; ${ }^{* *} \mathrm{p}=0.008$, Mann Whitney U-test. (F) Snapshots after $180 \mathrm{~s}$ of actin polymerization from TIRF movies recorded in the presence and absence of myosin-15 and ADP. Scale bar, $10 \mu \mathrm{m}$. (G) Quantification of F-actin density, indicative of nucleation rate, from TIRF movies. Solid lines and dashed lines / shading represent mean \pm S.D. at each time point; $N=3$. (H) Representative kymographs of elongating individual actin filaments from TIRF movies. (I) Quantification of filament elongation rates from TIRF movies. $\mathrm{N}=3$ independent replicates. Bars represent mean; $* * * * \mathrm{p}<0.0001$, one-way ANOVA. 
Actin alone<smiles>C1CCCCC1</smiles><smiles>C1CCCCC1</smiles><smiles>C1CCCCC1</smiles>

Actin + WT rigor myosin-15<smiles>C1CCCCCC1</smiles>

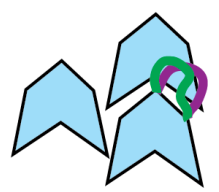

$$
\text { D-loop in + out }
$$
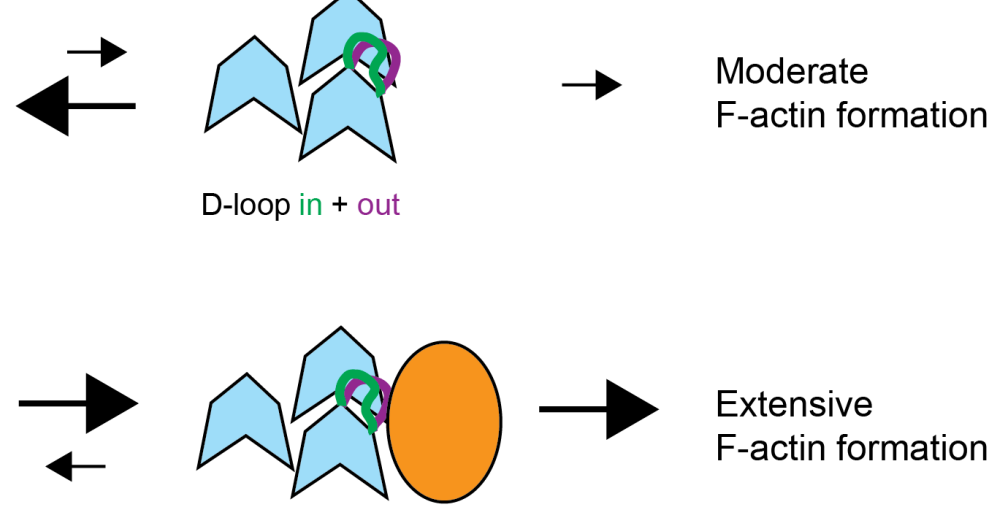

Extensive

F-actin formation
Very strong binding in rigor state stabilizes interprotomer contacts.
Flexibile D-loop supports

F-actin formation.

Actin + WT ADP myosin-15<smiles>C1CCCCC1</smiles><smiles>C1CCCCC1</smiles><smiles>C1CCCCC1</smiles>

Strong binding in ADP state stabilizes interprotomer contacts.
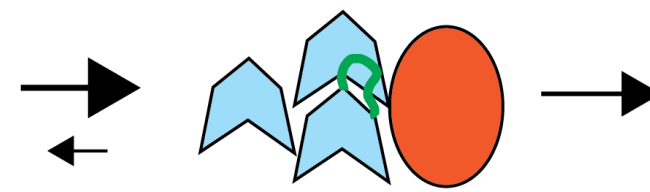

D-loop in
Intermediate

F-actin formation

Actin + jordan rigor myosin-15

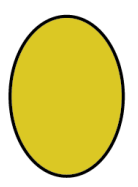

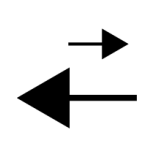

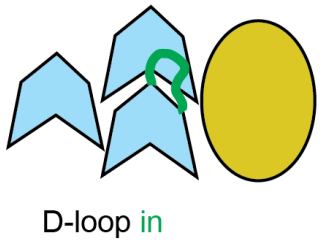

Rigid D-loop limits F-actin formation.<smiles>C1CCCCCC1</smiles><smiles>C1CCCCCC1</smiles>

Lower-affinity jordan mutant myosin-15 fails to stabilize interprotomer contacts.
Rigid D-loop further suppresses F-actin formation.

Figure 7. Schematic model for regulation of actin polymerization by myosin-15. We propose myosin15 enhances F-actin formation by stimulating nucleation, both by stabilizing actin-actin contacts and modulating D-loop structural plasticity. Myosin-15 nucleotide state facilitates tuning polymerizationstimulation activity, which could be harnessed for stereocilium height control. The jordan mutation disrupts F-actin regulation, thereby inhibiting polymerization. 
A

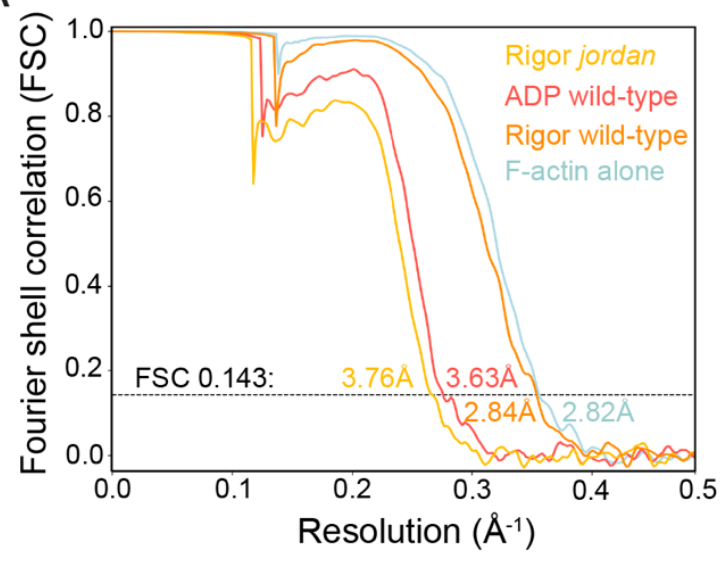

B

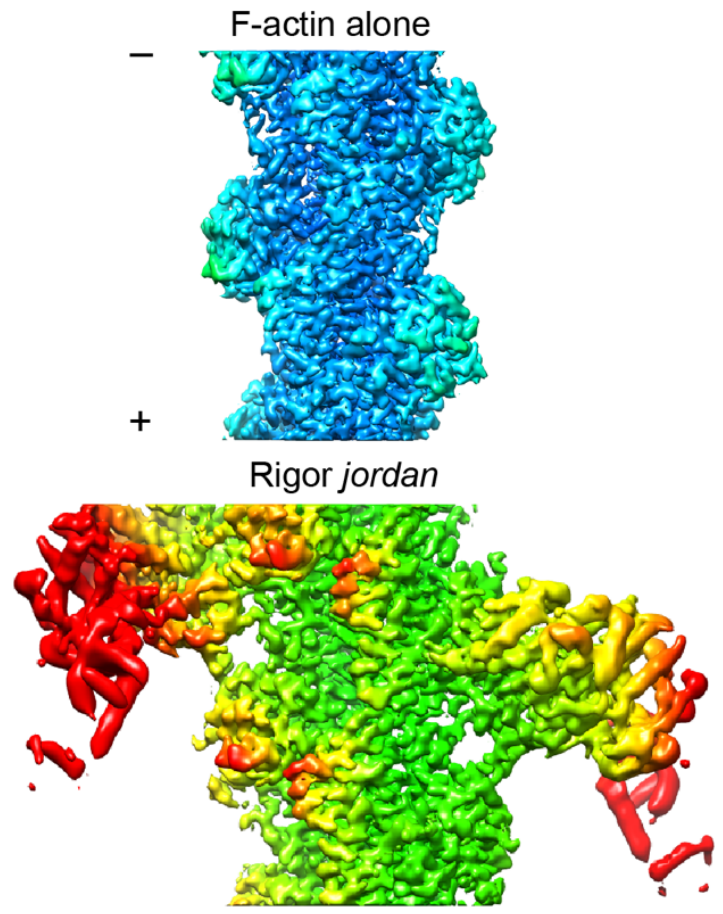

C

\begin{tabular}{|c|c|c|c|c|}
\hline Rise & $\begin{array}{c}\text { F-actin } \\
\text { alone }\end{array}$ & $\begin{array}{c}\text { Rigor } \\
\text { wild-type }\end{array}$ & $\begin{array}{c}\text { Rigor } \\
\text { jordan }\end{array}$ & $\begin{array}{c}\text { ADP } \\
\text { wild-type }\end{array}$ \\
\hline Twist & $-166.85^{\circ}$ & $-166.69^{\circ}$ & $-166.66^{\circ}$ & $-166.52^{\circ}$ \\
\hline
\end{tabular}
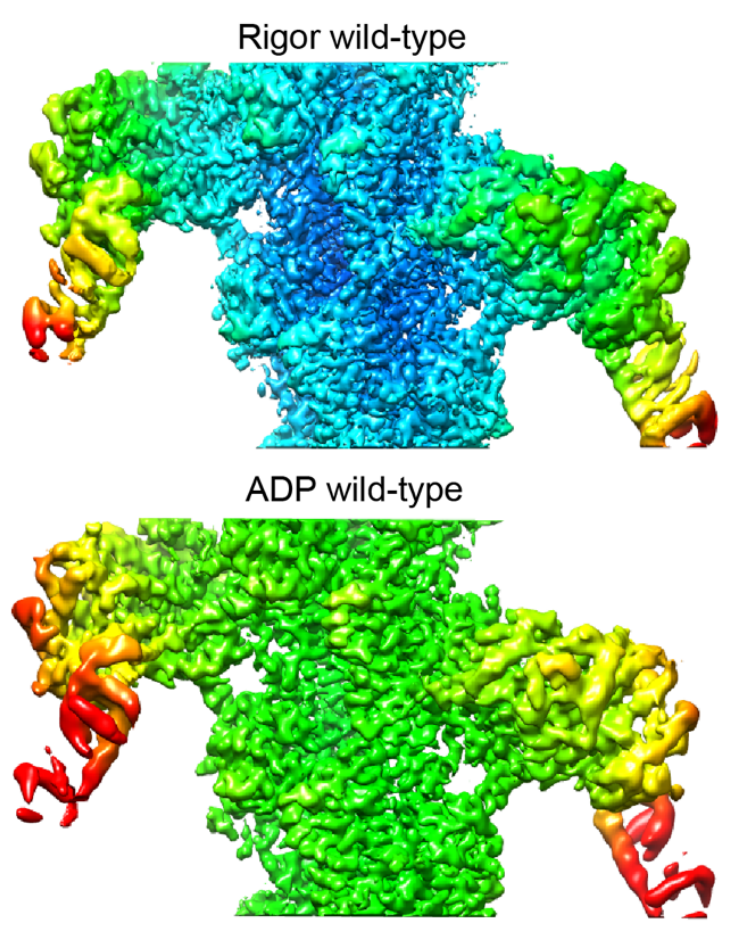

Local resolution:

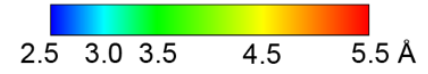

Figure S1. Resolution assessment of cryo-EM reconstructions and helical parameters. (A) Goldstandard Fourier shell correlation (FSC) curves for all 3D reconstructions presented in this study. Overall resolution is estimated by the FSC 0.143 criterion. (B) Local resolution estimation of the indicated reconstructions. (C) Helical parameters of the 3D reconstructions. 

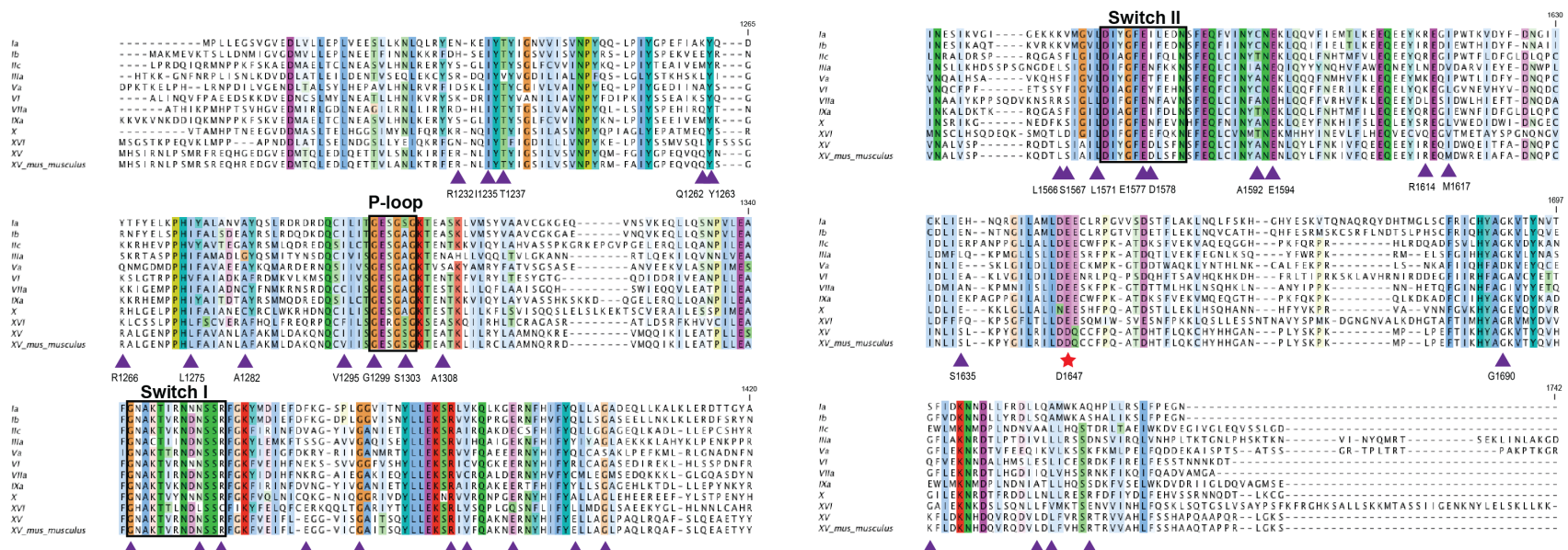

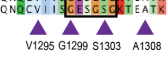

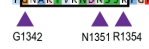

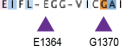
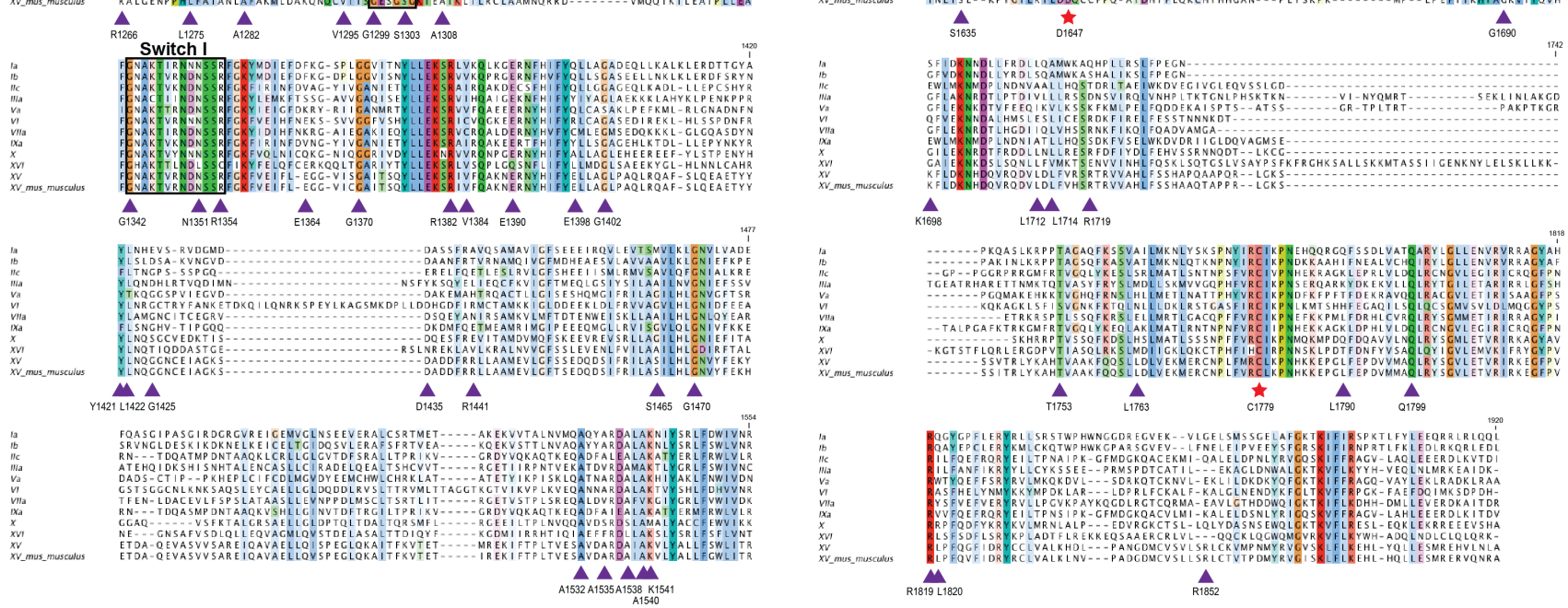

Figure S2. Sequence alignment of myosin motor domains. Aligned sequences correspond to: $H$. sapiens myosin-1a (NP_005370.1), $1 \mathrm{~b}$ (NP_001155291.1), 2c (NP_079005.3), 3a (NP_059129.3), 5a (NP_000250.3), 6 (NP_004990.3), 7a (NP_000251.3), 9a (NP_002464.1), 10 (NP_036466.2), 16 (NP_001185879.1), 15 (NP_057323.3), and M. musculus 15 (N̄P_874357). The alignment is colored by sequence conservation. The sites of D1647 and C1779 are indicated by red stars, and sites of deafness mutations are indicated by purple triangles. Sequence alignment was performed using Clustal Omega and formatted with Jalview. 
A

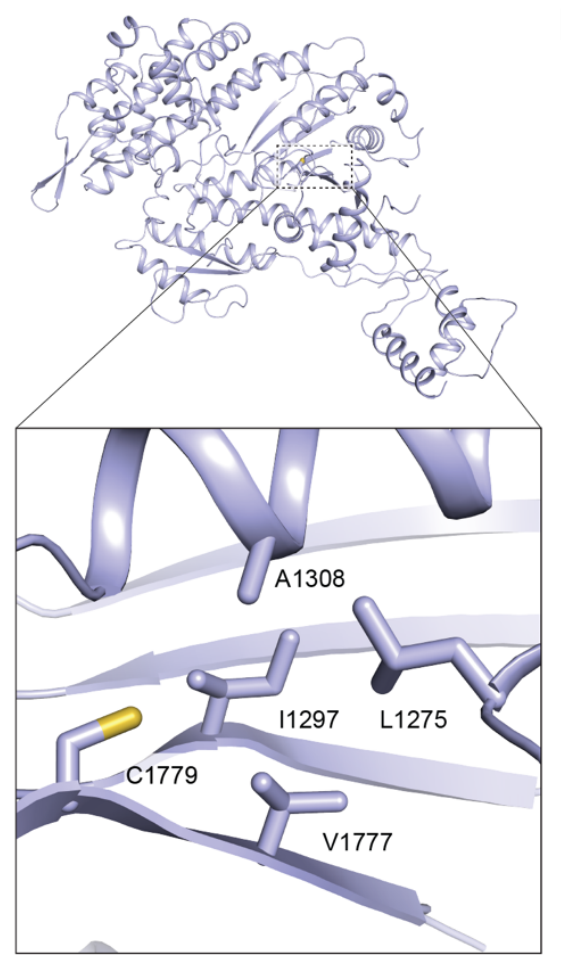

B

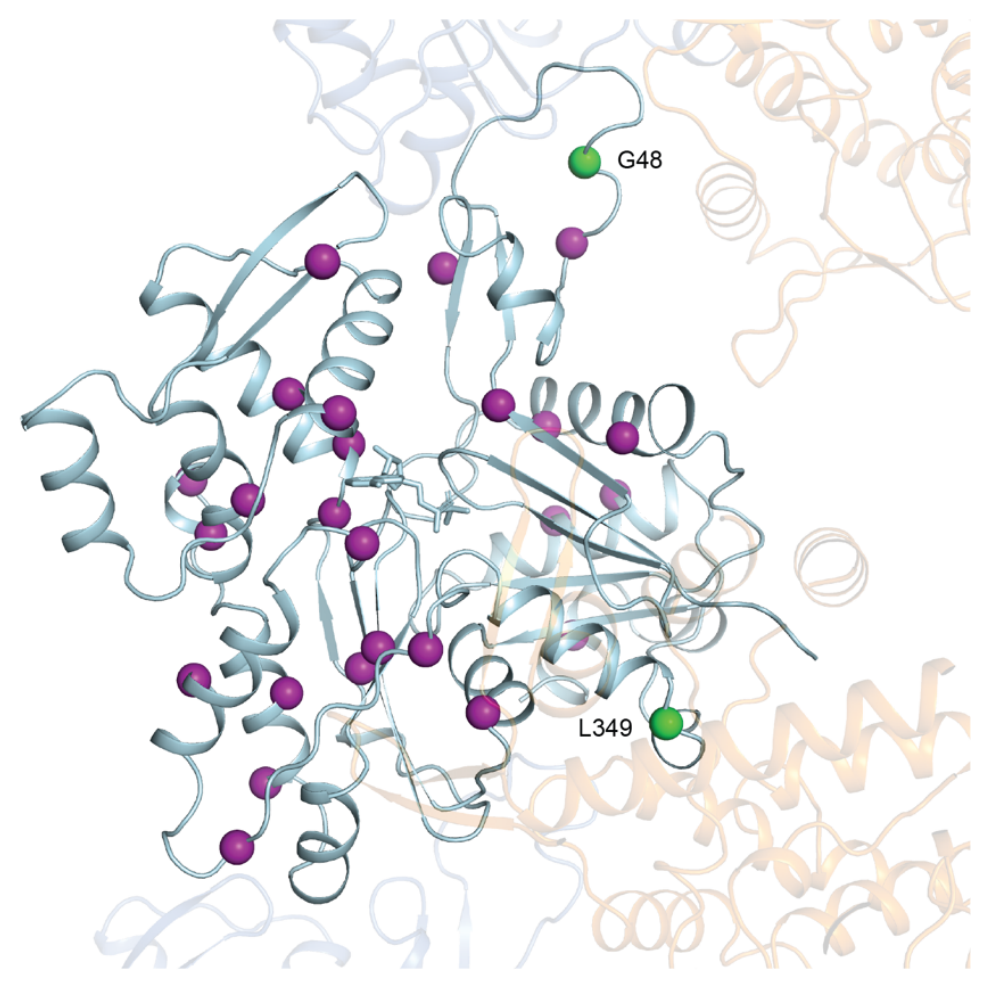

Figure S3. Additional structural analysis of deafness causing mutations. (A) The hydrophobic environment surrounding C1779, the site of the shaker 2 mutation. Hydrophobic residues are shown as sticks. (B) Deafness causing mutations on $\gamma$-actin shown as magenta spheres. Two residues located at the actomyosin-15 interface are highlighted in green. Adjacent actin subunits and myosin-15 are shown in transparent blue and orange, respectively. 
A
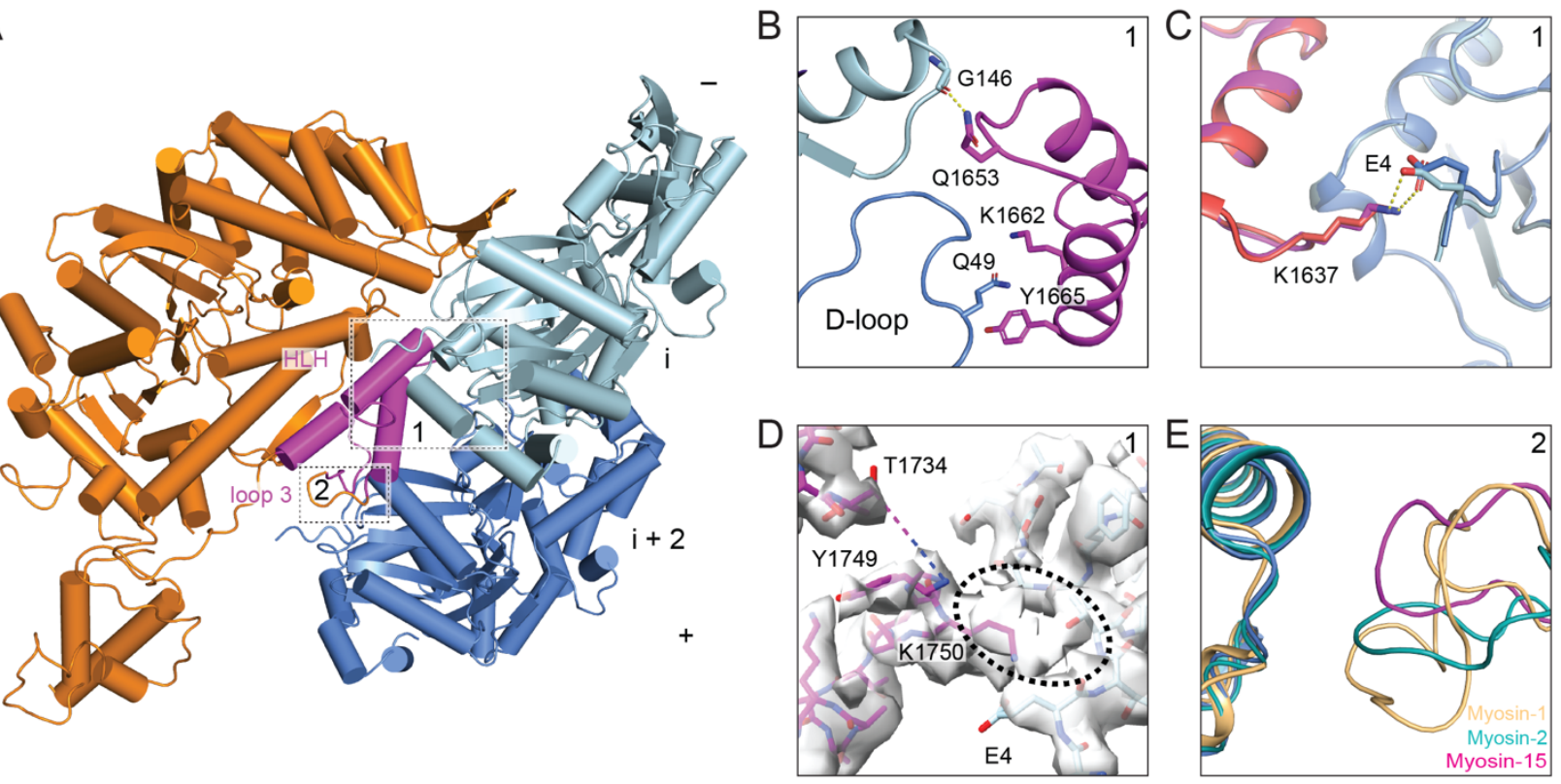

Figure S4. Additional interactions at the actomyosin-15 interface. (A) Atomic model of the interface between two actin subunits and rigor wild-type myosin-15 shown from an alternative perspective. Actin subunits i, i+2, and myosin-15 are colored in light blue, cornflower blue, and dark orange, respectively. The indicated actin interaction motifs are highlighted in magenta, and numbered boxes correspond to detail views in subsequent panels. (B) Electrostatic interactions between the HLH of rigor wild-type myosin-15 and F-actin. (C) Interaction between the activation loop of myosin-15 and the $\mathrm{N}$ terminus of actin subunit $i$. The HLH of the rigor and ADP wild-type myosin-15 are colored in magenta and deep salmon. The corresponding actin subunits are colored in light blue and cornflower blue. (D) The density map around loop 2 of rigor wild-type myosin-15. The models of myosin-15 and actin are colored in magenta and light blue. The disordered region of loop 2 between residues T1734 and Y1749 is represented by a dashed line. Extra density near the $\mathrm{N}$ terminus of actin is circled. (E) Structural comparison of the myosin loop 3-actin interface between rigor state myosin-15, myosin-1, and myosin-2. The structures are superimposed on the loop 3-interacting actin subunit. Loop 3 of myosin-15 is colored in magenta and the interacting actin is colored in cornflower blue, while loop 3 of myosin- 1 and myosin-2 are colored in light orange and teal, respectively, as are their interacting actin subunits. 
bioRxiv preprint doi: https://doi.org/10.1101/2021.07.09.451843; this version posted July 12, 2021. The copyright holder for this preprint (which was not certified by peer review) is the author/funder, who has granted bioRxiv a license to display the preprint in perpetuity. It is made available under aCC-BY-NC-ND 4.0 International license.

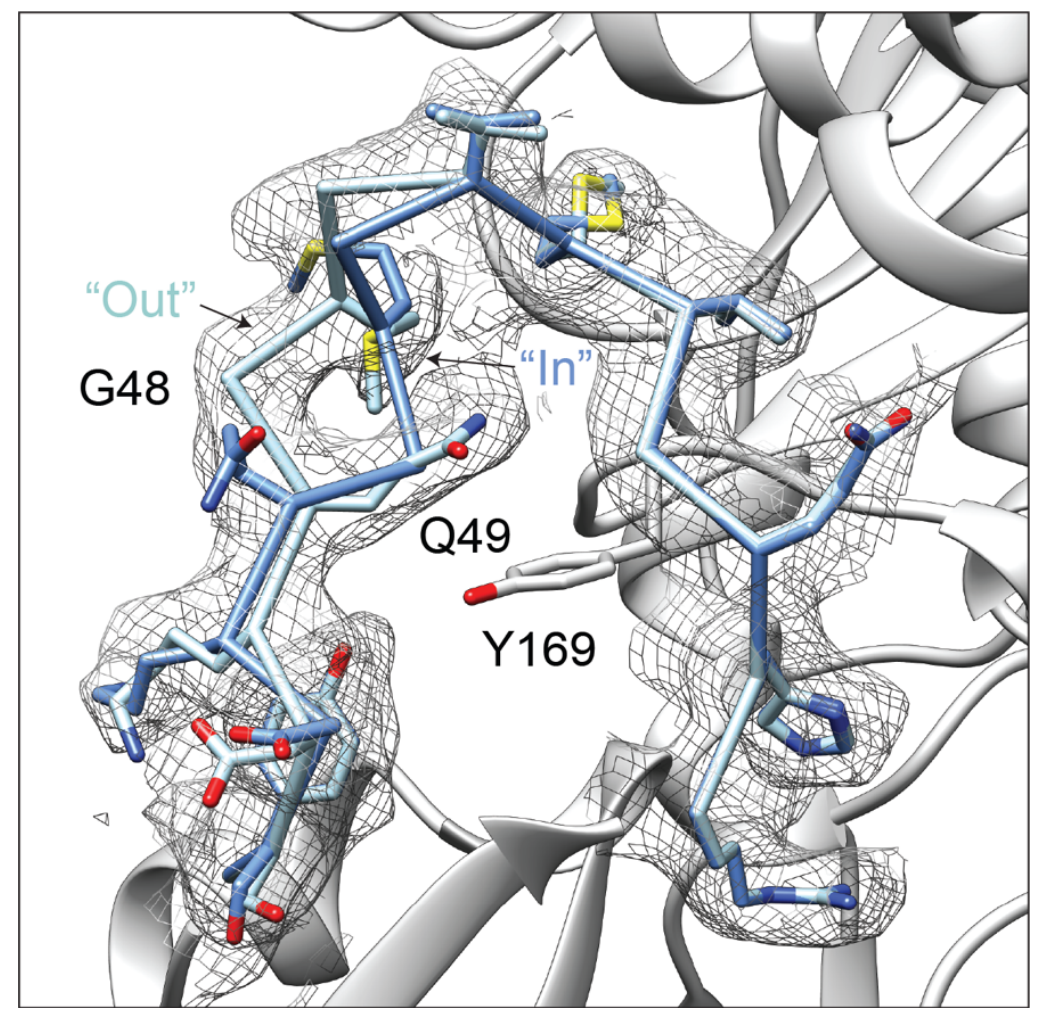

Figure S5. The D-loop of bare F-actin assumes two conformations. Magnified view of segmented cryo-EM density of the bare F-actin D-loop rendered at 0.025 RMS, superimposed with stick models of the "Out" and "In" D-loop conformations colored in light blue and cornflower blue, respectively. 

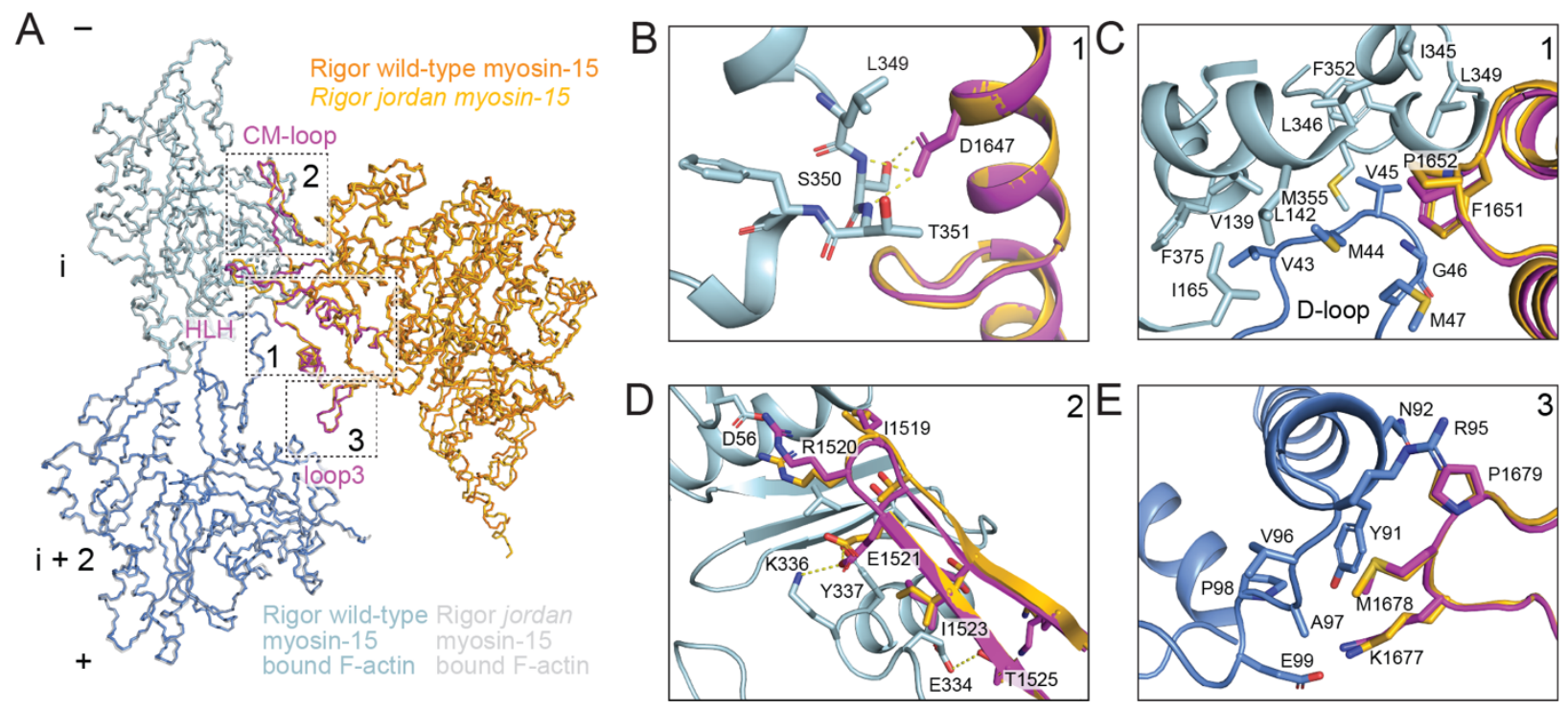

Figure S6. Structural comparison of rigor wild-type and jordan mutant actomyosin-15 at the actinmyosin interface. (A) Backbone representations of the rigor wild-type and jordan mutant actomyosin-15 models, superimposed on actin subunit i. Myosin-15's actin interacting motifs are highlighted in magenta on the rigor wild-type model. Numbered boxes correspond to detail views in subsequent panels, which match those displayed in Figure 3. (B) Although the D1647G lesion disrupts interactions in the jordan mutant, the overall HLH conformation in this region is maintained. (C-E) All other actin interface interactions mediated by the HLH (C), CM loop (D), and loop $3(\mathbf{E})$, are maintained in the jordan mutant. 

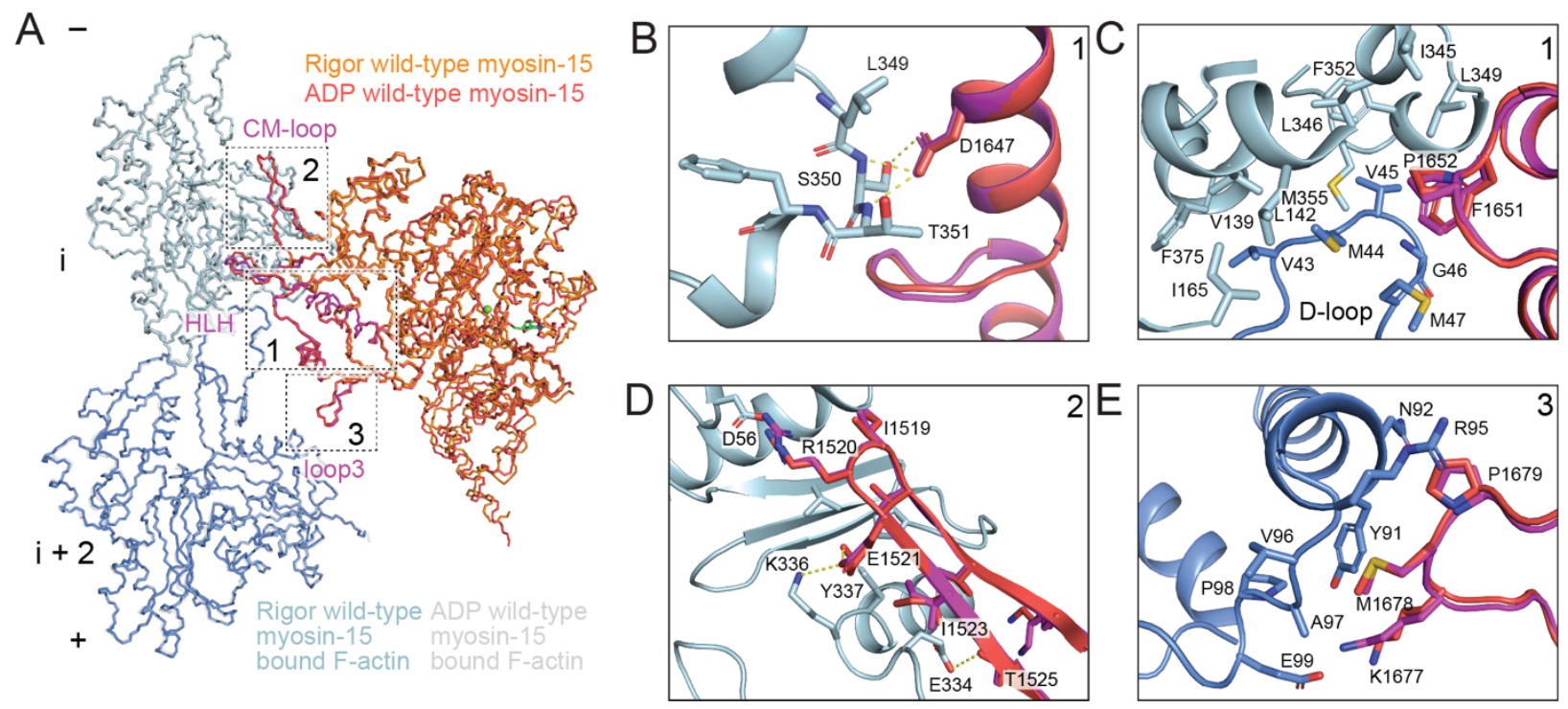

Figure S7. Structural comparison of rigor and ADP wild-type actomyosin-15 at the actin-myosin interface. (A) Backbone representations of the rigor and ADP wild-type actomyosin-15 models, superimposed on actin subunit i. Myosin-15's actin interacting motifs are highlighted in magenta on the rigor wild-type model. Numbered boxes correspond to detail views in subsequent panels, which match those displayed in Figure 3. (B-E) All actin interface interactions mediated by HLH residues D1647 (B) and F1651/P1652 (C), as well as the CM loop (D) and loop 3 (E), are present in both the ADP and rigor states. 
bioRxiv preprint doi: https://doi.org/10.1101/2021.07.09.451843; this version posted July 12, 2021. The copyright holder for this preprint (which was not certified by peer review) is the author/funder, who has granted bioRxiv a license to display the preprint in perpetuity. It is made available under aCC-BY-NC-ND 4.0 International license.

\begin{tabular}{|c|c|c|c|c|}
\hline & $\begin{array}{l}\text { F-actin alone } \\
\text { EMD-24321, PDB 7R8V }\end{array}$ & $\begin{array}{l}\text { Rigor WT myosin-15 } \\
\text { bound F-actin } \\
\text { EMD-24322, PDB 7R91 }\end{array}$ & $\begin{array}{l}\text { Rigor jordan myosin-15 } \\
\text { bound F-actin } \\
\text { EMD-24400, PDB 7RB9 }\end{array}$ & $\begin{array}{l}\text { ADP WT myosin-15 } \\
\text { bound F-actin } \\
\text { EMD-24399, PDB 7RB8 }\end{array}$ \\
\hline \multicolumn{5}{|c|}{ Data collection and processing } \\
\hline Microscope & Titan Krios & Titan Krios & Titan Krios & Titan Krios \\
\hline Voltage (kV) & 300 & 300 & 300 & 300 \\
\hline Detector & K2 Summit & K2 Summit & K2 Summit & K2 Summit \\
\hline Magnification & 29,000 & 29,000 & 29,000 & 29,000 \\
\hline Electron exposure $\left(\mathrm{e}^{-} / \AA^{2}\right)$ & 60 & 60 & 60 & 60 \\
\hline Exposure rate $\left(\mathrm{e}^{-} / \mathrm{pixel} / \mathrm{s}\right)$ & 1.5 & 1.5 & 1.5 & 1.5 \\
\hline Calibrated pixel size $(\AA)$ & 1.03 & 1.03 & 1.03 & 1.03 \\
\hline Defocus range $(\mu \mathrm{m})$ & -1.5 to -3.5 & -1.5 to -3.5 & -1.5 to -3.5 & -1.5 to -3.5 \\
\hline \multirow[t]{3}{*}{ Helical symmetry } & C1 & C1 & C1 & $\mathrm{C} 1$ \\
\hline & $27.19 \AA$ rise & $27.07 \AA$ rise & $27.08 \AA$ rise & $27.07 \AA$ rise \\
\hline & $-166.85^{\circ}$ twist & $-166.69^{\circ}$ twist & $-166.66^{\circ}$ twist & $-166.52^{\circ}$ twist \\
\hline $\begin{array}{l}\text { Initial filament segments } \\
\text { (no.) }\end{array}$ & 229,833 & 150,424 & 113,662 & 139,547 \\
\hline $\begin{array}{l}\text { Final filament segments } \\
\text { (no.) }\end{array}$ & 228,334 & 142,635 & 91,340 & 125,232 \\
\hline Map resolution $(\AA)$ & 2.82 & 2.83 & 3.76 & 3.63 \\
\hline FSC threshold & 0.143 & 0.143 & 0.143 & 0.143 \\
\hline \multicolumn{5}{|l|}{ Refinement } \\
\hline Initial model (PDB ID) & $6 \mathrm{BNO}$ & $6 \mathrm{BNO}$ & $6 \mathrm{BNO}$ & $6 \mathrm{BNO}$ \\
\hline Model resolution $(\AA)$ & 3.0 & 3.0 & 3.9 & 3.8 \\
\hline FSC threshold & 0.5 & 0.5 & 0.5 & 0.5 \\
\hline $\begin{array}{l}\text { Map sharpening B factor } \\
\left(\AA^{2}\right)\end{array}$ & -44.79 & -50.05 & -86.65 & -84.62 \\
\hline Model composition & 5 actin protomers & $\begin{array}{l}3 \text { actin protomers, } \\
1 \text { myosin-15 }\end{array}$ & $\begin{array}{l}3 \text { actin protomers, } \\
1 \text { myosin-15 }\end{array}$ & $\begin{array}{l}3 \text { actin protomers, } \\
1 \text { myosin-15 }\end{array}$ \\
\hline Non-hydrogen atoms & 14845 & 14320 & 14193 & 14226 \\
\hline Protein residues & 1855 & 1786 & 1786 & 1786 \\
\hline Ligands & $5 \mathrm{Mg} \cdot \mathrm{ADP}$ & $3 \mathrm{Mg} \cdot \mathrm{ADP}$ & $3 \mathrm{Mg} \cdot \mathrm{ADP}$ & 4 Mg.ADP \\
\hline \multicolumn{5}{|l|}{ B factors $\left(\AA^{2}\right)$} \\
\hline Protein & 37.16 & 54.6 & 120.24 & 79.79 \\
\hline Ligand & 27.66 & 29.25 & 77.38 & 70.69 \\
\hline \multicolumn{5}{|l|}{ R.M.S. deviations } \\
\hline Bond lengths $(\AA)$ & 0.004 & 0.004 & 0.004 & 0.004 \\
\hline Bond angles $\left({ }^{\circ}\right)$ & 0.97 & 0.978 & 0.979 & 1.018 \\
\hline \multicolumn{5}{|l|}{ Validation } \\
\hline MolProbity score & 1.44 & 1.37 & 1.7 & 1.84 \\
\hline Clash score & 5.56 & 5.01 & 9.24 & 8.73 \\
\hline Poor rotamers (\%) & 0 & 0.19 & 0 & 0.07 \\
\hline \multicolumn{5}{|l|}{ Ramachandran plot } \\
\hline Favored (\%) & 97.27 & 97.45 & 96.67 & 94.62 \\
\hline Allowed (\%) & 2.73 & 2.55 & 3.33 & 5.38 \\
\hline Disallowed (\%) & 0.00 & 0.00 & 0.00 & 0.00 \\
\hline
\end{tabular}

Table S1. Cryo-EM data collection, refinement, and validation statistics. 


\section{Supplementary Video Captions:}

Video S1: Morph of segmented actin density between F-actin alone and rigor wild-type myosin-15 bound F-actin. Maps were lowpass filtered to $5 \AA$.

Video S2: Detail view of D-loop in morph between F-actin alone and rigor wild-type myosin-15 bound F-actin. Maps are identical to those presented in Video S1.

Video S3: Morph of segmented actin density between rigor wild-type and jordan myosin-15 bound F-actin. Maps were lowpass filtered to $5 \AA$.

Video S4: Detail view of D-loop in morph between rigor wild-type and jordan myosin-15 bound Factin. Maps are identical to those presented in Video S3.

Video S5: Morph of segmented actin density between rigor and ADP wild-type myosin-15 bound F actin. Maps were lowpass filtered to $5 \AA$.

Video S6: Detail view of D-loop in morph between rigor and ADP wild-type myosin-15 bound Factin. Maps are identical to those presented in Video S5. 


\section{Methods:}

Expression and purification of myosin-15: Baculoviruses encoding either the wild-type, or jordan variant of the mouse myosin-15 motor domain (NP_874357.2, aa. 1 - 743) truncated after the $2^{\text {nd }} \mathrm{IQ}$ domain, and including a C-terminal EGFP and FLAG moiety, were produced as described (Moreland, 2021). Briefly, Sf9 cells were seeded at $2 \times 10^{6}$ cells / mL in ESF-921 (Expression Systems), and infected simultaneously with myosin-15 baculovirus at a multiplicity of infection (MOI) of 5. Additional dualpromoter baculoviruses expressing bovine smooth muscle essential (MYL6) and chicken regulatory (MYL12B) light chains $(\mathrm{MOI}=5)$, as well as mouse UNC45B and HSP90AA1 $(\mathrm{MOI}=5)$ were included (Bird et al., 2014; Pato et al., 1996). Cells were harvested 48 - 72 hours post-infection and flash frozen in liquid nitrogen.

Myosin-15 motor domains were purified as described (Moreland, 2021). Briefly, cells were homogenized in Extraction Buffer: 10 mM MOPS, $500 \mathrm{mM} \mathrm{NaCl}, 1 \mathrm{mM}$ EGTA, $10 \mathrm{mM} \mathrm{MgCl}$, 2 mM ATP, $0.2 \mathrm{mM}$ PMSF, $0.1 \mathrm{mM}$ DTT, $1 \mathrm{mM} \mathrm{NaN}_{3}, 2 \mu \mathrm{g} \cdot \mathrm{mL}^{-1}$ leupeptin, 1 x protease inhibitor cocktail (Halt EDTA-free; Thermo Scientific), pH 7.2. Lysates were clarified at 48,000 x $g$ for 30 minutes and incubated with FLAG M2 affinity resin (Sigma-Aldrich) for 3 hours at $4{ }^{\circ} \mathrm{C}$. The FLAG resin was then washed in High-Salt Buffer (10 mM MOPS, $500 \mathrm{mM} \mathrm{NaCl}, 1 \mathrm{mM}$ EGTA, $5 \mathrm{mM} \mathrm{MgCl} 2,1 \mathrm{mM} \mathrm{ATP}, 0.1$ mM PMSF, $0.1 \mathrm{mM}$ DTT, $1 \mathrm{mM} \mathrm{NaN}, 2 \mu \mathrm{g} \cdot \mathrm{mL}^{-1}$ leupeptin, $\mathrm{pH}$ 7.2) followed by Low-Salt Buffer (10 mM MOPS, $60 \mathrm{mM} \mathrm{NaCl}, 1 \mathrm{mM}$ EGTA, $0.1 \mathrm{mM}$ PMSF, $0.1 \mathrm{mM} \mathrm{DTT}, 1 \mathrm{mM} \mathrm{NaN}, 2 \mu \mathrm{g} \cdot \mathrm{mL}^{-1}$ leupeptin, $\mathrm{pH}$ 7.0). Myosin-15 protein was subsequently eluted with $0.2 \mathrm{mg} \cdot \mathrm{mL}^{-1} 3 \mathrm{x}$ FLAG peptide (American Peptide, CA) in Low-Salt Buffer. Eluted myosin-15 motor domain was then purified by anion exchange chromatography (5/50 MonoQ GL; Cytiva). After injecting the sample, the column was washed with $10 \mathrm{mM}$ MOPS, $100 \mathrm{mM} \mathrm{NaCl}, 1 \mathrm{mM}$ EGTA, $0.1 \mathrm{mM}$ PMSF, 1 mM DTT, pH 7.0, then eluted with a linear gradient to $1 \mathrm{M} \mathrm{NaCl}$. Fractions eluting at $\sim 31 \mathrm{mS} \cdot \mathrm{cm}^{-1}$ were concentrated $(10,000 \mathrm{MWCO})$ and further purified by size exclusion chromatography (Superdex 200, Cytiva) with isocratic elution in 10 mM MOPS, $100 \mathrm{mM} \mathrm{KCl,} 0.1 \mathrm{mM}$ EGTA, $1 \mathrm{~mm} \mathrm{NaN}$, $0.1 \mathrm{mM}$ PMSF, $1 \mathrm{mM} \mathrm{DTT}, 1 \mu \mathrm{g} \cdot \mathrm{mL}^{-1}, \mathrm{pH}$ 7.0. The myosin-15 : ELC : RLC complex $(1: 1: 1)$ eluted as a single peak, and was concentrated $(10,000$ 
MWCO) before determining concentration $\left(\mathrm{A} 280, \varepsilon=88,020 \mathrm{M}^{-1} \cdot \mathrm{cm}^{-1}\right)$. Purity was confirmed by SDSPAGE (4-20\% TGX, BioRad).

Actin purification: Chicken skeletal muscle actin was purified as previously described (MacLeanFletcher and Pollard, 1980) and stored in G-Mg buffer: $2 \mathrm{mM}$ Tris-HCl pH 8.0, 0.5 mM DTT, $0.2 \mathrm{mM}$ $\mathrm{MgCl}_{2}, 0.01 \% \mathrm{NaN}_{3}$ at $4{ }^{\circ} \mathrm{C}$. F-actin was prepared by mixing $5 \mu \mathrm{M}$ monomeric actin with KMEI buffer (50 mM KCl, $1 \mathrm{mM} \mathrm{MgCl} 2,1 \mathrm{mM}$ EGTA, $10 \mathrm{mM}$ imidazole $\mathrm{pH}$ 7.0) supplemented with $0.01 \% \mathrm{NP} 40$ substitute (Roche). The mixture was incubated at room temperature for 1 hour to stimulate polymerization, followed by $4^{\circ} \mathrm{C}$ overnight.

Grid preparation: For the F-actin alone sample, $3 \mu \mathrm{l}$ of F-actin diluted to $0.6 \mu \mathrm{M}$ in KMEI + NP40 was applied to a glow discharged C-flat 1.2/1.3 holey carbon Au 300 mesh grid (Electron Microscopy Sciences) in a Leica GP plunge freezer operating at $25^{\circ} \mathrm{C}$. After $60 \mathrm{~s}$ incubation, the grid was blotted from the back with Whatman no. 5 filter paper for $4 \mathrm{~s}$ and flash frozen in liquid ethane. To prepare the rigor wild-type and jordan mutant myosin- 15 bound F-actin samples, $10 \mathrm{U} / \mathrm{ml}$ apyrase was added to $6 \mu \mathrm{M}$ myosin-15 and incubated on ice for 20 minutes to remove nucleotide. $60 \mathrm{~s}$ after applying $3 \mu \mathrm{l}$ of $0.6 \mu \mathrm{M}$ F-actin to a glow discharged grid, $3 \mu 1$ of myosin- 15 was added and incubated for $60 \mathrm{~s}$. After that $3 \mu 1$ of the mixture solution was removed and another $3 \mu \mathrm{l}$ of myosin- 15 was applied. After an additional $60 \mathrm{~s}$ of incubation, $3 \mu \mathrm{l}$ of the mixture solution was removed and the grid was blotted for $4 \mathrm{~s}$ and flash frozen in liquid ethane. The ADP-Mg state wild-type myosin-15 bound F-actin specimen was prepared identically, except that wild-type myosin-15 was incubated with $5 \mathrm{mM}$ ADP-Mg for 20 minutes prior to grid preparation.

Cryo-EM data collection: Data were collected on a FEI Titan Krios operating at $300 \mathrm{kV}$ equipped with a Gatan K2-summit detector using super-resolution mode. Exposures were targeted using stage translations with a single exposure per hole using the SerialEM software suite (Mastronarde, 2005), and movies were 
recorded at a nominal magnification of $29,000 \mathrm{X}$, corresponding to a calibrated pixel size of $1.03 \AA$ at the specimen level (super-resolution pixel size of $0.515 \AA$ / pixel). Each exposure was fractionated across 40 frames with a total electron dose of $60 \mathrm{e}^{-/} \AA^{2}\left(1.5 \mathrm{e}^{-} / \AA^{2} /\right.$ frame $)$ and a total exposure time of $10 \mathrm{~s}$, with defocus values ranging from -1.5 to $-3.5 \mu$ m underfocus.

Cryo-EM image processing: Image processing was carried out using the Iterative Helical Real Space Refinement (IHRSR) protocol as implemented in the RELION 3.0 pipeline (Egelman, 2007; Zivanov et al., 2018). Unless otherwise noted, all steps were carried out in RELION. Movie stacks were motion corrected, dose weighted and summed with 2 x 2 binning (to a $1.03 \AA$ pixel size) using the MotionCor2 algorithm (Zheng et al., 2017) with 5 x 5 patches as implemented in RELION. Contrast Transfer Function (CTF) estimation was performed with CTFFIND4 (Rohou and Grigorieff, 2015). Filaments were autopicked and split into overlapping segments with a step size of $81 \AA$, corresponding to 3 actin protomers, then extracted in a box size of 512 pixels and subjected to two-dimensional (2D) classification. Segments contributing to featureful class-averages were selected and subjected to three-dimensional (3D) classification with 4 classes, initialized with helical parameters of $27 \AA$ rise and $-166.7^{\circ}$ twist. The cryoEM maps of F actin alone (EMD-7115) and myosin VI bound F actin (EMD-7116) were low pass filtered to $35 \AA$ to serve as the initial models for $\mathrm{F}$ actin alone and myosin- 15 decorated $\mathrm{F}$ actin datasets, respectively. The best $3 \mathrm{D}$ class from each dataset was selected and low-pass filtered to $35 \AA$ to serve as the initial reference for subsequent 3D auto-refinement. Segments contributing to all the 3D classes, which did not substantially vary in features, were included for 3D auto-refinement. After 3D autorefinement, post-processing was performed for each dataset using a 3D mask with a $\mathrm{Z}$ length of $50 \%$ of the box size, resulting in maps with $4.23 \AA$ (actin alone), $4.23 \AA$ (rigor wild-type myosin-15), $6.16 \AA$ (rigor jordan myosin-15), and 5.56 A (ADP wild-type myosin-15) resolution based on the Fourier shell correlation (FSC) 0.143 criterion.

To further improve the resolution, iterative CTF refinement, Bayesian polishing and 3D autorefinement was carried out by adapting a recently described procedure (Mei et al., 2020). Briefly, CTF 
refinement was initially performed without beam-tilt estimation, followed by another round of 3D-autorefinement using the converged map from the last refinement as the initial reference, low-pass filtered to $35 \AA$. Subsequent post-processing was performed using a 3D mask with a $Z$ length of $30 \%$ of the box size, then a second round of CTF refinement with beam-tilt estimation, Bayesian polishing and 3D autorefinement using the converged map from the previous refinement low-pass filtered to $35 \AA$ as the initial reference. A final masked refinement was performed for the rigor wild-type reconstruction using the $30 \%$ $\mathrm{Z}$ length mask, which modestly improved the resolution: masked refinement did not improve either of the other actomyosin reconstructions. Final post-processing for each dataset was performed with a $30 \% \mathrm{Z}$ length mask, leading to final resolution assessments of $2.82 \AA$ (F-actin alone), $2.83 \AA$ (rigor wild-type myosin-15), $3.76 \AA$ (rigor jordan mutant myosin-15), and $3.63 \AA$ (ADP wild-type myosin-15) by the FSC 0.143 criterion (Figure S1). Local resolution estimation and filtering was performed using RELION 3.0. Data acquisition and processing details are listed in Table S1.

Model building and structure refinement: For F-actin alone, a published actin model (PDB: 6BNO) (Gurel et al., 2017) was docked into the EM density by rigid body fitting in UCSF Chimera (Pettersen et al., 2004) and manually adjusted in Coot (Emsley and Cowtan, 2004). In this starting model, the D-loop was modeled in the "Out" conformation. For the actomyosin structures, an initial homology model of myosin-15 was generated with I-TASSER (Zhang, 2008) using the crystal structure of myosin V (PDB:1OE9) (Coureux et al., 2003) as the template. The myosin-15 model and the F-actin alone model were then docked into each cryo-EM density map to generate actomyosin starting models.

Models were then subjected to Rosetta density-guided model rebuilding (DiMaio et al., 2015). For each initial model, 200 models were generated, and the top 10 lowest energy models were manually inspected in Coot. Non-overlapping stretches of amino acids that fit the cryo-EM density best were selected from these individual models then stitched together to build the full model. For F-actin alone and rigor wild-type actomyosin-15, the D-loop of a subset of the 10 lowest-energy models adopted the "In" conformation, which were subsequently used to model this conformer for these states. D-loops for all 
lowest-energy models in the jordan mutant reconstruction and wild-type ADP reconstruction adopted the "In" conformation. The stitched full models were refined using phenix.real_space.refine in the Phenix software package (Afonine et al., 2018), iterated with manual adjustment in Coot. Model validation was conducted with MolProbity (Williams et al., 2018) as implemented in Phenix.

Molecular graphics and multiple sequence analysis: Figures and movies were generated with PyMol (The PyMOL Molecular Graphics System, Version 2.3.4; Schrödinger, LLC), UCSF Chimera (Pettersen et al., 2004), and ChimeraX (Goddard et al., 2018). Multiple sequence alignment was performed with Clustal Omega (Madeira et al., 2019) and formatted with Jalview (Waterhouse et al., 2009).

Pyrene actin polymerization: Skeletal rabbit actin was purified and labeled on Cys 374 with N-(1pyrene)-iodoacetamide (Criddle et al., 1985). A correction factor was applied for determining pyrene actin concentration, $\mathrm{A}_{\text {corr }}=\mathrm{A}_{290}-\left(0.127 * \mathrm{~A}_{344}\right)$. Actin polymerization was measured in a cuvette-based fluorometer (PTI Quantamaster 400, HORIBA Scientific) with excitation at $365 \mathrm{~nm}$ and emission at 407 nm. Gel filtered G-actin monomers (10\% pyrene labeled) were desalted (PD SpinTrap G-25, Cytiva) into a modified G-buffer without ATP (2 mM Tris-HCl, $0.1 \mathrm{mM} \mathrm{CaCl}_{2}, 1 \mathrm{mM} \mathrm{NaN}_{3}, 1 \mathrm{mM}$ DTT, pH 8.0), stored on ice and used within 3 hours. G-actin was converted to the $\mathrm{Mg}^{2+}$ bound form by addition of 50 $\mu \mathrm{M} \mathrm{MgCl}_{2}$ and $0.2 \mathrm{mM}$ EGTA for two minutes at room temperature, before starting the polymerization reaction by mixing G-actin (3 x stock) with KMEI buffer (1.5x stock) in a 1:2 ratio, respectively. Wildtype myosin-15 and ADP were included in the $1.5 \mathrm{x}$ KMEI buffer, as needed. Final reaction conditions were $2 \mu \mathrm{M}$ G-actin, $1 \mu \mathrm{M}$ myosin-15, $50 \mathrm{mM} \mathrm{KCl}, 1 \mathrm{mM} \mathrm{MgCl} 2,1 \mathrm{mM}$ EGTA, $10 \mathrm{mM}$ imidazole, $\mathrm{pH}$ 7.0 at $25 \pm 0.1^{\circ} \mathrm{C}$. Data were corrected for the reaction dead-time.

Single-filament actin polymerization: Skeletal rabbit actin was purified, labeled on Cys 374 using tetramethylrhodamine-5-maleimide (Adipogen Life Sciences), and its concentration measured using $\mathrm{A}_{\text {corr }}$ $=\mathrm{A}_{290}-\left(0.208 * \mathrm{~A}_{550}\right)$ (Fujiwara et al., 2002). Biotinylated skeletal muscle actin (\#8109-01, HyperMol, 
Germany) was dialyzed against G-buffer and cleared by ultracentrifugation at 100,000 x $g$ for 1 hour. Gactin stocks were prepared with TMR-actin (20\%) and biotin-actin (10\%) doping, and desalted into a modified ATP-free G-buffer as described above. Cover glass $(24 \times 50 \mathrm{~mm}$, \#1.5, Marienfeld Superior, Germany) were sequentially sonicated in 2\% Hellmanex III (Hellma, Germany), 1 M KOH, $100 \%$ ethanol, and finally Milli-Q water, prior to drying under a nitrogen stream and processing under argon plasma (ZEPTO, Diener Electronic, Germany). Cleaned cover glass were coated with mPEG-silane (2 mg / mL, Laysan Bio, AL) and biotin-PEG-silane $\left(2 \mu \mathrm{g} \cdot \mathrm{mL}^{-1}\right.$, Laysan Bio) diluted in $96 \%$ ethanol, $0.1 \%$ (v/v) $\mathrm{HCl}$, and baked at $70{ }^{\circ} \mathrm{C}$ for 1 hour. Cover slips were rinsed in $96 \%$ ethanol, sonicated, followed by rinsing and sonication in Milli-Q water, and finally dried under a filtered nitrogen stream. Flow chambers were assembled using double-sided sticky tape to create a channel on a glass slide. Flow cells were washed with T50 buffer (10 mM Tris· $\mathrm{HCl}, 50 \mathrm{mM} \mathrm{KCl,} 1 \mathrm{mM}$ DTT, $\mathrm{pH}$ 8.0), and incubated with $0.1 \mathrm{mg}$ / $\mathrm{mL}$ neutravidin (Thermo Scientific) in T50 for 1 minute, then washed in $1 \mathrm{mg} / \mathrm{mL}$ bovine serum albumin (A0281, Sigma Aldrich) in T50 for $30 \mathrm{~s}$, followed by a final wash of T50.

Experiments were performed in the following buffer: $50 \mathrm{mM} \mathrm{KCl}, 1 \mathrm{mM} \mathrm{MgCl} 2,1 \mathrm{mM}$ EGTA, $10 \mathrm{mM}$ imidazole, $10 \mathrm{mM}$ DTT, $15 \mathrm{mM}$ glucose, $0.5 \%$ methylcellulose, $20 \mu \mathrm{g} \cdot \mathrm{mL}^{-1}$ catalase, $100 \mu \mathrm{g} \cdot \mathrm{mL}^{-}$ ${ }^{1}$ glucose oxidase, $\mathrm{pH} 7.0$ at $21 \pm 1{ }^{\circ} \mathrm{C}$. Purified myosin- 15 motor domain $(1 \mu \mathrm{M})$ and ADP were included as needed. The reaction flow cell was imaged using an inverted microscope (Nikon Ti-E2) equipped with an oil immersion objective (CFI Apochromat TIRF 100x, 1.49 N.A., Nikon) for objective-style total internal reflection fluorescence (TIRF) microscopy (H-TIRF, Nikon). TMR fluorescence was excited using $561 \mathrm{~nm}$, and emission filtered (ET630/75m, Chroma). Images were captured on an EM-CCD camera (iXon Ultra 888, Andor) controlled by NIS-Elements (AR version 5.2, Nikon). Data were corrected for the assay dead-time. Images were background subtracted and registered (descriptor-based series registration, $2 \mathrm{~d} / 3 \mathrm{~d}+\mathrm{t}$ ) in FIJI (https://fiji.sc). Actin filament densities were quantified using the Analyze Particle command (size $>3$ pixel $^{2}$, circularity: 0.0-1.0) to count particles within a $50 \times 50 \mu \mathrm{m}$ region of interest (ROI) that was randomly selected from the image. A minimum of 3 experiments, from 
two independent protein preparations, were analyzed for each condition. Filament elongation rates were calculated using kymographs generated in Elements Software (Nikon).

\section{Methods Reference:}

Afonine, P.V., Poon, B.K., Read, R.J., Sobolev, O.V., Terwilliger, T.C., Urzhumtsev, A., and Adams, P.D. (2018). Real-space refinement in PHENIX for cryo-EM and crystallography. Acta Crystallogr D Struct Biol 74, 531-544.

Bird, J.E., Takagi, Y., Billington, N., Strub, M.P., Sellers, J.R., and Friedman, T.B. (2014). Chaperoneenhanced purification of unconventional myosin 15 , a molecular motor specialized for stereocilia protein trafficking. Proc Natl Acad Sci U S A 111, 12390-12395.

Coureux, P.D., Wells, A.L., Menetrey, J., Yengo, C.M., Morris, C.A., Sweeney, H.L., and Houdusse, A. (2003). A structural state of the myosin V motor without bound nucleotide. Nature 425, 419-423.

Criddle, A.H., Geeves, M.A., and Jeffries, T. (1985). The use of actin labelled with N-(1pyrenyl)iodoacetamide to study the interaction of actin with myosin subfragments and troponin/tropomyosin. Biochem J 232, 343-349.

DiMaio, F., Song, Y., Li, X., Brunner, M.J., Xu, C., Conticello, V., Egelman, E., Marlovits, T., Cheng, Y., and Baker, D. (2015). Atomic-accuracy models from 4.5-A cryo-electron microscopy data with density-guided iterative local refinement. Nat Methods 12, 361-365.

Egelman, E.H. (2007). The iterative helical real space reconstruction method: surmounting the problems posed by real polymers. J Struct Biol 157, 83-94.

Emsley, P., and Cowtan, K. (2004). Coot: model-building tools for molecular graphics. Acta Crystallogr D Biol Crystallogr 60, 2126-2132.

Fujiwara, I., Takahashi, S., Tadakuma, H., Funatsu, T., and Ishiwata, S. (2002). Microscopic analysis of polymerization dynamics with individual actin filaments. Nat Cell Biol 4, 666-673.

Goddard, T.D., Huang, C.C., Meng, E.C., Pettersen, E.F., Couch, G.S., Morris, J.H., and Ferrin, T.E. (2018). UCSF ChimeraX: Meeting modern challenges in visualization and analysis. Protein Science 27, $14-25$.

Gurel, P.S., Kim, L.Y., Ruijgrok, P.V., Omabegho, T., Bryant, Z., and Alushin, G.M. (2017). Cryo-EM structures reveal specialization at the myosin VI-actin interface and a mechanism of force sensitivity. Elife 6 .

MacLean-Fletcher, S., and Pollard, T.D. (1980). Identification of a factor in conventional muscle actin preparations which inhibits actin filament self-association. Biochem Biophys Res Commun 96, 18-27.

Madeira, F., Park, Y.M., Lee, J., Buso, N., Gur, T., Madhusoodanan, N., Basutkar, P., Tivey, A.R.N., Potter, S.C., Finn, R.D., et al. (2019). The EMBL-EBI search and sequence analysis tools APIs in 2019. Nucleic Acids Res 47, W636-W641. 
Mastronarde, D.N. (2005). Automated electron microscope tomography using robust prediction of specimen movements. J Struct Biol 152, 36-51.

Mei, L., Espinosa de Los Reyes, S., Reynolds, M.J., Leicher, R., Liu, S., and Alushin, G.M. (2020). Molecular mechanism for direct actin force-sensing by alpha-catenin. Elife 9 .

Moreland, Z.G., Jiang, F., Aguilar, C., Barzik, M., Gong, R., Shams, A., Faaborg-Andersen, C., Werth, J.C., Harley, R., Sutton, D.C., Cole, S.M., Parker, A., Morse, S., Wilson, E., Takagi, Y., Sellers, J.R., Brown, S.D.M., Friedman, T.B., Alushin, G.M., Bowl M.R., Bird, J.E. (2021). Myosin-driven Nucleation of Actin Filaments Drives Stereocilia Development Critical for Hearing. In preparation.

Pato, M.D., Sellers, J.R., Preston, Y.A., Harvey, E.V., and Adelstein, R.S. (1996). Baculovirus expression of chicken nonmuscle heavy meromyosin II-B. Characterization of alternatively spliced isoforms. J Biol Chem 271, 2689-2695.

Pettersen, E.F., Goddard, T.D., Huang, C.C., Couch, G.S., Greenblatt, D.M., Meng, E.C., and Ferrin, T.E. (2004). UCSF Chimera-A visualization system for exploratory research and analysis. Journal of Computational Chemistry 25, 1605-1612.

Rohou, A., and Grigorieff, N. (2015). CTFFIND4: Fast and accurate defocus estimation from electron micrographs. J Struct Biol 192, 216-221.

Waterhouse, A.M., Procter, J.B., Martin, D.M., Clamp, M., and Barton, G.J. (2009). Jalview Version 2--a multiple sequence alignment editor and analysis workbench. Bioinformatics 25, 1189-1191.

Williams, C.J., Headd, J.J., Moriarty, N.W., Prisant, M.G., Videau, L.L., Deis, L.N., Verma, V., Keedy, D.A., Hintze, B.J., Chen, V.B., et al. (2018). MolProbity: More and better reference data for improved all-atom structure validation. Protein Sci 27, 293-315.

Zhang, Y. (2008). I-TASSER server for protein 3D structure prediction. BMC Bioinformatics 9, 40.

Zheng, S.Q., Palovcak, E., Armache, J.P., Verba, K.A., Cheng, Y., and Agard, D.A. (2017). MotionCor2: anisotropic correction of beam-induced motion for improved cryo-electron microscopy. Nat Methods 14, 331-332.

Zivanov, J., Nakane, T., Forsberg, B.O., Kimanius, D., Hagen, W.J., Lindahl, E., and Scheres, S.H. (2018). New tools for automated high-resolution cryo-EM structure determination in RELION-3. Elife 7. 